\title{
CHANGES IN SPECIES COMPOSITION AND LITTER DYNAMICS ALONG A FRAGMENT SIZE GRADIENT IN SUBTROPICAL BROADLEAVED FORESTS OF MEGHALAYA, NORTHEAST INDIA
}

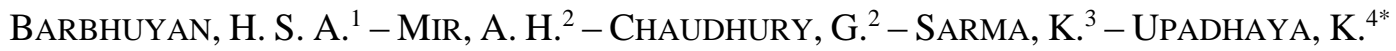 \\ ${ }^{I}$ Department of Botany, School of Life Sciences, North-Eastern Hill University, Shillong \\ 793 022, India
}

${ }^{2}$ Department of Environmental Studies, School of Human and Environmental Sciences, NorthEastern Hill University, Shillong 793 022, India

${ }^{3}$ University School of Environment Management, Guru Gobind Singh Indraprastha University, New Delhi 110078, India

${ }^{4}$ Department of Basic Sciences and Social Sciences, School of Technology, North-Eastern Hill University, Shillong 793 022, India

*Corresponding author

e-mail: upkri@yahoo.com

(Received $8^{\text {th }}$ Jan 2021; accepted $14^{\text {th }}$ May 2021)

\begin{abstract}
Habitat fragmentation creates numerous small forest patches separated by human land use that may affect the compositional and structural attributes and ecosysem functions particularly the nutrient dynamics in forest ecosystems. We tested this hypothesis along a fragment size gradient in subtropical broadleaved forests in Cherrapunjee area of Meghalaya, northeast India following standard methods of vegetation sampling and nutrient dynamics. The study revealed that the structure and composition of woody species, shrubs and herbs varied significantly $(\mathrm{p}<0.05)$ along a fragment size gradient. Similarly, the leaf litter standing crop as well as total litter fall and annual $\mathrm{N}$ and $\mathrm{P}$ return through litter fall varied significantly $(\mathrm{p}<0.05)$ across fragments and were higher in the larger fragments as compared to the smaller fragments. Therefore, the results of the study collectively suggest that understanding the overall ecological implications of the effect of fragmentation on the structure and functioning of subtropical broadleaved forest ecosystem of Meghalaya, north east India, is imperative to outline pertinent conservation strategies. Keywords: ecosystem function, nutrient dynamics, plant diversity, stand structure, litterfall
\end{abstract}

\section{Introduction}

Throughout the world, habitat loss and fragmentation have been considered the primary cause for biodiversity loss and ecosystem degradation (Laurance and Bierregaard, 1997; Laurance and Peres, 2006; Wu, 2013; Wilson et al., 2016). The process of fragmentation can be explained as a biphasic event, characterized by the reduction of the total amount of forest area, followed by loss of habitat area, changes in the spatial configuration of the landscape, resulting in the isolation of smaller patches (Wilcove et al., 1986; Saunders et al., 1991; Didham et al., 2012). These processes cause differences not only in species diversity but also in composition between fragments and continuous forests.

The physical and biological changes associated with forest fragmentation include habitat loss and insularization (Lovejoy et al., 1986; Laurance, 1990), reduction in the number of species and changes in plant dynamics owing to difference in the forest 
spatial organization such as the size, shape, and isolation of fragments, edge effects, invasion of foreign species, and other disturbances (Murcia, 1995; Laurance et al., 2002; Hobbs and Yates, 2003). Fragmentation leads to community disassembly following decline of both species abundance and diversity (Turner and Corlett, 1996; Tabarelli et al., 1999; Echeverría et al., 2007) and alters the ability of the remnant forests to maintain their original biodiversity and ecological processes (Echeverría et al., 2007). Fragment size has been reported to be the major determinant of changes in woody plant composition (Tabarelli et al., 1999; Rosati et al., 2010; Fahrig, 2013; Pao and Upadhaya, 2017). Fragmentation has short- and long- term effects on species composition (Harper et al., 2005; Oliveira et al., 2008; Santos et al., 2008; Laurance et al., 2011; Andrade et al., 2015), that often favor the proliferation of generalist and pioneer species (Melo et al., 2007; Laurance and Vasconcelos, 2009; Chabrerie et al., 2013) and hardy exotic species (Laurance and Vasconcelos, 2009; Carmo et al., 2011; Chabrerie et al., 2013; Lambert et al., 2014). Small patches are often exposed to a greater disturbance (Pao and Upadhaya, 2017; Schmidt, 2019) and are prone to a large number of gaps that eventually affect tree regeneration and recruitment (Lovejoy et al., 1986; Laurance et al., 1998). Given the role of biodiversity in the provision of ecosystem services, a reduced resistance (or higher susceptibility) to the increasing frequency and intensity of anthropogenic disturbance is likely to cause widespread degradation of forests and the provision of such ecosystem services (Brockerhoff et al., 2017).

The consequences of habitat fragmentation on biodiversity are well documented (Fahrig, 2003) and there is a general consensus on the strong negative impact that fragmentation has on biodiversity within forests (Sala et al., 2000). Most of the studies that investigated the effects of habitat fragmentation have typically focused on changes in the population or community dynamics of the flora and fauna (Laurance and Bierregaard, 1997), while its effect on ecosystem functioning (viz., nutrient cycling and organic matter decomposition) are limited (Didham, 1998; Vasconcelos and Luizão, 2004; Rubinstein and Vasconcelos, 2005; Vasconcelos and Laurance, 2005; Lindsay and Cunningham, 2009). Fragmentation of habitats is known to cause changes in the community composition as well as the ecosystem functions in fragmented landscapes (Turner and Corlett, 1996; Laurance and Cochrane, 2001; Cochrane and Laurance, 2002) as manifested by alteration of nutrient dynamics following shift in composition of dominant species and microclimatic buffering in the fragments (Saunders et al., 1991; Murcia, 1995; Fahrig, 2003; Haddad et al., 2015).

Among the various ecosystem functions, litter dynamics plays an important role in regulating plant community composition through its effects on recruitment (Facelli and Pickett, 1991; Hastwell and Facelli, 2000) and nutrient cycle (Vitousek, 2004). Litter deposited on the forest floor acts as an input-output system of nutrients and acts as a temporary sink for nutrients and functions as 'a slow release nutrient source' (White, 1988), thereby ensuring a permanent input of nutrients to the soil (Cuevas and Medina, 1988). However, it is the rates at which litter falls and decays that are the main regulators of primary productivity, energy flow and nutrient cycling in forest ecosystems (Bray and Gorham, 1964; Swift et al., 1981; Prescott et al., 2004; Prescott, 2010). Feeley (2004) observed that fragmentation can cause significant changes in the quantity of accumulated litter and strongly influence the rate at which nutrients are mineralized and made available to plants.

The state of Meghalaya, situated in the north eastern part of India, lies between $24^{\circ} 58^{\prime} \mathrm{N}$ to $26^{\circ} 07^{\prime} \mathrm{N}$ latitude and $89^{\circ} 48^{\prime} \mathrm{E}$ to $92^{\circ} 51^{\prime} \mathrm{E}$ longitude and covers an area of 
$22,429 \mathrm{~km}^{2}$, which accounts to $0.68 \%$ of the total geographical area of the country (ISFR, 2019). The state is a part of the 'Indo-Burma' global biodiversity hotspots (Mittermeier et al., 2004). The forest cover of the state in the year 2019 was $76.33 \%$. However, during the period 2017-2019, the state has lost a forest area of $23 \mathrm{~km}^{2}$ (ISFR, 2019) due to a number of human activities such as overexploitation, deforestation, shifting cultivation, mining and urbanization (Pao and Upadhaya, 2017). The current vulnerabilities of the forest systems in Meghalaya arise from forest disturbances and fragmentation. Thus, it is important to address the effects of fragmentation on community structure and ecosystem functioning. It is hypothesized that fragmentation modulates species composition and their abundance in forest patches of different size that would affect nutrient dynamics on the forest floor by influencing resource quality and quantity of above ground litter and nutrient reservoir. This in turn would have an effect on the availability of nutrients on the forest floor. Therefore, the objectives of the present study were to investigate the effect of fragmentation on (i) species diversity and community structure and (ii) nutrient dynamics through the study of accumulation of Nitrogen $(\mathrm{N})$ and Phosphorous (P) by litter in subtropical broadleaved forests.

\section{Methods}

\section{Study sites}

The present study was undertaken in Cherrapunjee and its adjoining areas in the state of Meghalaya, northeast India (Figure 1). The vegetation of the area is classified as subtropical broadleaved forest (Champion and Seth, 1968) and represents the remnants of the climax forest of the area. They are dense evergreen forest with short stature and the tree height rarely exceeds $20 \mathrm{~m}$. The climate of the area is tropical monsoonal and is directly influenced by the southwest monsoon moving from the Bay of Bengal. It has a distinct hot-wet and cold-dry period. The wet period extends from May to October, during which more than $80 \%$ of the total rainfall occurs. The dry period prevails from November to March with <22 mm rainfall. The mean annual rainfall varies from 8000$10000 \mathrm{~mm}$ and the average temperature ranges from $1.7^{\circ} \mathrm{C}$ to $24^{\circ} \mathrm{C}$. The soils are derived from gneissic complex parent materials; they are dark brown to dark reddishbrown in colour, varying in depth from 50-200 cm. The texture of soils varies from loamy to sandy loam. The reaction of the soils varies from acidic $(\mathrm{pH} 5.0$ to 6.0) to strongly acidic ( $\mathrm{pH} 4.5$ to 5.0 ), rich in organic carbon and nitrogen but deficient in phosphorus and potassium (http://megagriculture.gov.in/PUBLIC/agri_scenario_soil.aspx).

To assess the impact of fragmentation on species richness, density and population structure of woody species in the subtropical broadleaved forest, a total of fourteen forest fragments ranging in size from 2.01 to $>100$ ha were selected for the present study. These fragments were grouped into five classes viz. Very small $(<3 \mathrm{ha})$, Small (4-6 ha), Medium (8-10 ha), Large (17-86 ha) and Very Large (>100 ha). For each fragment class three replicate forest patches were selected. However, due to unavailability of continuous forests in the study area, two replicated plots were maintained from a single Very Large fragment. These replicated sites were abbreviated as Very small (VS1, VS2, VS3), Small (S1, S2, S3), Medium (M1, M2, M3), Large (L1, L2, L3) and Very Large (VL1, VL2), respectively (Table 1). All the fragments were once a part of the same forest. 


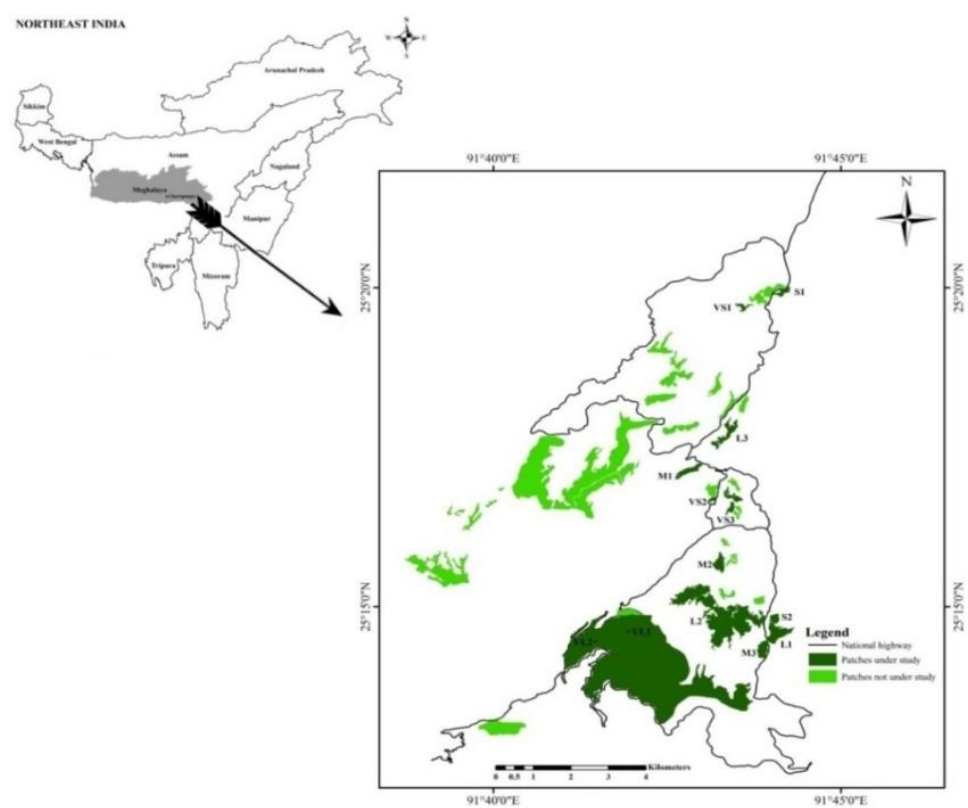

Figure 1. Map showing the location of the study area at Cherrapunjee in Meghalaya, northeast India

\section{Plant diversity sampling}

The vegetation was assessed by laying a belt transect of $250 \mathrm{~m}$ length $\times 20 \mathrm{~m}$ width in each of the selected fragments. The transects were further divided into, quadrats of $10 \mathrm{~m} \times 10 \mathrm{~m}$ for inventorizations of all woody species having a minimum diameter at breast height (dbh) of $\geq 5 \mathrm{~cm}$, quadrats of $5 \mathrm{~m} \times 5 \mathrm{~m}$ for shrubs and quadrats of $2 \mathrm{~m} \times 2 \mathrm{~m}$ for herbs. Plant samples were collected and identified with the help of regional floras (Kanjilal et al., 1940; Haridasan and Rao, 1987). The Herbaria at Botanical Survey of India (ASSAM), Eastern Regional Circle, Shillong and Department of Botany, NorthEastern Hill University (NEHU), Shillong were also consulted for correct identification of the specimens. The nomenclature of the species follows the regional flora. The field data was analyzed for number of species and quantitative community parameters such as frequency, density, basal area and importance value index (IVI) were computed following Misra (1968) and Mueller-Dombois and Ellenberg (1974). Various diversity indices such as Shannon-Wiener, Simpson and Pielou were calculated following Magurran (1988) as follows:

Shannon-Wiener index of diversity:

$$
H^{\prime}=-\sum p i \ln p i
$$

where, $\mathrm{H}^{\prime}$ is the measure of diversity and pi is the proportion of the total sample belonging to the $\mathrm{i}^{\text {th }}$ species.

Simpson's dominance index:

$$
D=\sum p i^{2}
$$

where, D is the Simpson index of dominance and pi is the proportional individuals of species $i$ in the community. 
Pielou's evenness index:

$$
E=\frac{H^{\prime}}{\ln S}
$$

where, E is Pielou's evenness index, $\mathrm{H}^{\prime}$ is the Shannon-Wiener index of diversity and $\mathrm{S}$ is the number of species in the community

\section{Litter dynamics}

\section{Forest floor Litter}

Standing crop of litter expressed as the dry mass per unit ground area at a given time $\left(\mathrm{g} \mathrm{m}^{-2}\right)$ was determined at monthly intervals from May 2015-April 2017 by randomly laying ten $1 \mathrm{~m} \times 1 \mathrm{~m}$ size quadrats in each studied forest fragment. Litter samples were collected (cleared and swept of any deposited debris) from all the quadrats following the litter sampling procedures by Spain (1984). The litter was then brought to the laboratory and segregated into leaf, woody and miscellaneous (including flowers, fruits and other unidentified plant detritus) fractions. The segregated litter fractions were oven dried at $60^{\circ} \mathrm{C}$ to a constant weight and expressed as the litter accumulation or standing crop of litter.

\section{Litterfall}

Freshly fallen litter was collected from 10 randomly located $1 \mathrm{~m} \times 1 \mathrm{~m}$ permanent quadrats at monthly interval to determine aboveground litter production from May 2015April 2017. The litter present in each quadrat was collected and expressed as the dry mass per unit ground area over a period of one month $\left(\mathrm{g} \mathrm{m}^{-2} \mathrm{month}^{-1}\right)$. Annual production was expressed as the sum of all positive increments $\left(\mathrm{g} \mathrm{m}^{-2} \mathrm{yr}^{-1}\right)$ during each sampling period. The litter samples were brought to the laboratory and segregated into leaf, woody and miscellaneous fractions and oven dried at $60^{\circ} \mathrm{C}$ to a constant weight. Samples of each fraction of the litter were ground, homogenized and passed through a $0.2 \mathrm{~mm}$ mesh for determination of nitrogen $(\mathrm{N})$ and phosphorus $(\mathrm{P})$ contents according to the micro-Kjeldahl method (Allen et al., 1974). All chemical analyses were carried out in triplicate. The nutrient content $\left(\mathrm{kg} \mathrm{ha}^{-1}\right)$ of each litter component was estimated by multiplying the mean concentrations of each mineral-element $\left(\mathrm{g} \mathrm{kg}^{-1}\right)$ in each litter components with the mean mass of the respective litter component $\left(\mathrm{kg} \mathrm{ha}^{-1}\right)$ for each site and expressed as the potential nutrient input.

\section{Data analysis}

The data for ecosystem functional attributes such as forest floor litter, total litterfall, and nutrient ( $\mathrm{N}$ and $\mathrm{P}$ ) accumulation through litter fall were measured on monthly basis and were pooled seasonally and presented fragment wise. The analysis considered two factors: fragment size (between subjects) and litter fractions (within subject) with four levels (autumn, winter, spring and rainy) spread across two years. Assumptions of ANOVA were met through test for normality of variables (Kolmogorov-Smirnov test), and homogeneity of group variances (Levene's test). The relationships of fragment size with vegetation characteristics and litter dynamics were analyzed using linear regression model. All statistical analysis was performed using the software SPSS, version 20.0. 


\section{Results}

\section{Floristic composition, species richness and diversity}

A total of 225 woody species belonging to 127 genera and 65 families were enumerated from all the studied fragments (Table 1). Species richness, density and basal area of woody ( $\geq 5 \mathrm{~cm} \mathrm{dbh}$ ) species varied along the fragment size gradient (Table 1 and Figure 2). The woody species richness in the fragments ranged from 50 species in VS1 to 78 in VL1 (Table 1) of which there were 77 species that did not re-occur in more than one forest fragment, whereas, two species namely Helicia nilagirica Bedd. and Castanopsis tribuloides (Sm.) DC. occurred in all the studied fragments. The dominant family was Lauraceae ( 27 species) followed by Rubiaceae (14 species), Rosaceae (13 species), Fagaceae ( 9 species) and Fabaceae ( 8 species). There were 29 families that were represented by one species and nine families by two species each.

Shannon-Wiener's diversity index $\left(\mathrm{H}^{\prime}\right)$ for woody species ranged from 3.09 to 3.72 across the studied forest fragments. Similarly, Simpson dominance index (D) ranged from 0.01 to 0.08 . The corresponding Pielou's evenness index ranged from 0.22-0.87 across the studied forest fragments (Table 1).

The species richness of shrubs in the fragments showed a reverse trend to that of woody species and ranged from 33 species in VL1 to 52 in S1 (Table 1 and Figure 2). A total of 216 shrubs belonging to 92 genera and 65 families were enumerated from all the studied fragments. Among shrubs the dominant family included Rubiaceae, Lamiaceae, Asteraceae, Primulaceae, Ericaceae, Menispermaceae, Rosaceae, Symplocaceae and Urticaceae. Similarly, the species richness of herbs increased from 40 species in S1 to 58 in VL1 thus exhibiting similarity to woody species along the fragment size gradient (Table 1 and Figure 2) with a total of 232 herbs belonging to 169 genera and 74 families. The dominant family of herbaceous species in the studied fragments included Poaceae, Asteraceae, Rubiaceae, Polypodiaceae, Araceae, Orchidaceae, Piperaceae, Polygonaceae, Fabaceae and Rosaceae.

\section{Stand density and basal area}

The density of woody individuals in the fragments increased along the fragment size gradient and ranged from 560 individuals $\mathrm{ha}^{-1}$ in VS1 to 1220 individuals ha ${ }^{-1}$ in VL2 (Table 1 and Figure 2) with mean density of 758 individuals $\mathrm{ha}^{-1}$ in VS, 949 individuals $\mathrm{ha}^{-1}$ in S, 978 individuals ha ${ }^{-1}$ in M, 1107 individuals $\mathrm{ha}^{-1}$ in $\mathrm{L}$ and 1161 individuals ha ${ }^{-1}$ in VL fragment classes. In terms of density, the dominant species in VS1 was Quercus kamroopii whereas Castanopsis tribuloides was the dominant species in VS2 and VS3, S, M1, M2, L1 and VL2. The dominant species was Syzygium cuneatum in M3, Castanopsis kurzii, in L2, Helicia nilagirica in L3 and Syzygium tetragonum in VL1. The basal area increased along the fragment size gradient from $26.33 \mathrm{~m}^{2} \mathrm{ha}^{-1}$ in S3 to $59.3 \mathrm{~m}^{2}$ $\mathrm{ha}^{-1}$ in VL2 (Table 1 and Figure 2). The mean basal area $\left(\mathrm{m}^{2} \mathrm{ha}^{-1}\right)$ was $37.92 \mathrm{~m}^{2} \mathrm{ha}^{-1}$ in VS, $28.9 \mathrm{~m}^{2} \mathrm{ha}^{-1}$ in $\mathrm{S}, 27.37 \mathrm{~m}^{2} \mathrm{ha}^{-1}$ in $\mathrm{M}, 40.48 \mathrm{~m}^{2} \mathrm{ha}^{-1}$ in $\mathrm{L}$ and $49.41 \mathrm{~m}^{2} \mathrm{ha}^{-1}$ in VL classes, respectively.

The shrubs and herbs density are shown in Table 1. While, the density of shrubs decreased along the fragment size gradient from 10280 individuals ha ${ }^{-1}$ in VS2 to 6464 individuals ha ${ }^{-1}$ in VL2 (Table 1, Figure 2), the density of herbs showed an increase from 226600 individuals ha ${ }^{-1}$ in VS1, to 250600 individuals ha ${ }^{-1}$ in VL2 fragment class (Table 1 and Figure 2). 
Table 1. Vegetation characteristics in different fragment categories

\begin{tabular}{|c|c|c|c|c|c|c|c|c|c|c|c|c|c|c|c|}
\hline \multirow{3}{*}{ Habit } & \multirow{3}{*}{ Parameters } & \multicolumn{14}{|c|}{ Fragment category } \\
\hline & & \multicolumn{3}{|c|}{ Very Small } & \multicolumn{3}{|c|}{ Small } & \multicolumn{3}{|c|}{ Medium } & \multicolumn{3}{|c|}{ Large } & \multicolumn{2}{|c|}{ Very Large } \\
\hline & & VS1 & VS2 & VS3 & S1 & S2 & S3 & M1 & M2 & M3 & L1 & L2 & L3 & VL1 & VL2 \\
\hline \multirow{8}{*}{$\begin{array}{c}\text { Woody } \\
\text { species }(\geq \\
5 \mathrm{~cm} \mathrm{dbh})\end{array}$} & No. of genera & 39 & 52 & 48 & 38 & 43 & 43 & 41 & 49 & 50 & 54 & 53 & 53 & 64 & 53 \\
\hline & No. of species & 50 & 68 & 60 & 55 & 56 & 55 & 53 & 67 & 65 & 71 & 77 & 72 & 78 & 77 \\
\hline & Density $\left(\mathrm{ha}^{-1}\right)$ & 560 & 806 & 908 & 974 & 982 & 890 & 902 & 994 & 1038 & 1026 & 1076 & 1220 & 1161 & 1202 \\
\hline & $\begin{array}{c}\text { Basal area }\left(\mathrm{m}^{2}\right. \\
\left.\mathrm{ha}^{-1}\right)\end{array}$ & 54.54 & 29.47 & 29.75 & 33.11 & 26.33 & 27.26 & 25 & 29.35 & 27.76 & 31.61 & 38.93 & 52.06 & 39.53 & 59.3 \\
\hline & $\begin{array}{c}\text { Shannon- } \\
\text { Wiener index }\end{array}$ & 3.29 & 3.47 & 3.09 & 3.25 & 3.52 & 2.6 & 3.063 & 3.48 & 3.42 & 3.52 & 3.38 & 3.56 & 3.56 & 3.72 \\
\hline & $\begin{array}{c}\text { Simpson } \\
\text { dominance } \\
\text { index }\end{array}$ & 0.06 & 0.06 & 0.12 & 0.08 & 0.04 & 0.19 & 0.11 & 0.05 & 0.06 & 0.04 & 0.06 & 0.05 & 0.08 & 0.03 \\
\hline & $\begin{array}{c}\text { Pielou's } \\
\text { evenness index }\end{array}$ & 0.84 & 0.82 & 0.75 & 0.81 & 0.87 & 0.64 & 0.77 & 0.82 & 0.83 & 0.82 & 0.78 & 0.83 & 0.87 & 0.87 \\
\hline & $\begin{array}{c}\text { Disturbance } \\
\text { index }\end{array}$ & 55 & 36 & 32 & 31 & 22 & 30 & 41 & 46 & 47 & 18 & 47 & 21 & 13 & 22 \\
\hline \multirow{4}{*}{ Shrubs } & $\begin{array}{l}\text { No. of species } \\
\text { Density }\left(\mathrm{ha}^{-1}\right)\end{array}$ & $\begin{array}{c}51 \\
10264\end{array}$ & $\begin{array}{c}51 \\
10280\end{array}$ & $\begin{array}{c}46 \\
6560\end{array}$ & $\begin{array}{c}52 \\
10080\end{array}$ & $\begin{array}{c}48 \\
9144\end{array}$ & $\begin{array}{c}51 \\
8032\end{array}$ & $\begin{array}{c}48 \\
9496\end{array}$ & $\begin{array}{c}49 \\
8152\end{array}$ & $\begin{array}{c}49 \\
7544\end{array}$ & $\begin{array}{c}36 \\
6480\end{array}$ & $\begin{array}{c}45 \\
6568\end{array}$ & $\begin{array}{c}43 \\
6496\end{array}$ & $\begin{array}{c}33 \\
5144\end{array}$ & $\begin{array}{c}35 \\
6464\end{array}$ \\
\hline & $\begin{array}{c}\text { Shannon } \\
\text { Wiener Index }\end{array}$ & 3.83 & 3.57 & 3.13 & 3.68 & 3.43 & 3.54 & 3.32 & 3.72 & 3.7 & 3.27 & 3.61 & 3.26 & 3.19 & 3.22 \\
\hline & $\begin{array}{c}\text { Simpson } \\
\text { dominance } \\
\text { index }\end{array}$ & 0.03 & 0.04 & 0.07 & 0.03 & 0.04 & 0.04 & 0.05 & 0.03 & 0.03 & 0.05 & 0.03 & 0.06 & 0.05 & 0.05 \\
\hline & $\begin{array}{c}\text { Pielou's } \\
\text { evenness index }\end{array}$ & 0.97 & 0.91 & 0.82 & 0.93 & 0.89 & 0.9 & 0.86 & 0.95 & 0.95 & 0.91 & 0.95 & 0.87 & 0.91 & 0.91 \\
\hline \multirow{4}{*}{ Herbs } & $\begin{array}{l}\text { No. of species } \\
\text { Density }\left(\text { ha }^{-1}\right)\end{array}$ & $\begin{array}{c}46 \\
226600\end{array}$ & $\begin{array}{c}54 \\
299600\end{array}$ & $\begin{array}{c}47 \\
199800\end{array}$ & $\begin{array}{c}40 \\
201600\end{array}$ & $\begin{array}{c}44 \\
252400\end{array}$ & $\begin{array}{c}47 \\
225000\end{array}$ & $\begin{array}{c}50 \\
200800\end{array}$ & $\begin{array}{c}53 \\
202000\end{array}$ & $\begin{array}{c}57 \\
254000\end{array}$ & $\begin{array}{c}49 \\
246200\end{array}$ & $\begin{array}{c}55 \\
211600\end{array}$ & $\begin{array}{c}44 \\
217800\end{array}$ & $\begin{array}{c}58 \\
203200\end{array}$ & $\begin{array}{c}54 \\
250600\end{array}$ \\
\hline & $\begin{array}{c}\text { Shannon } \\
\text { Wiener Index }\end{array}$ & 3.66 & 3.44 & 3.34 & 3.31 & 3.42 & 3.49 & 3.51 & 3.72 & 3.79 & 3.55 & 3.77 & 3.33 & 3.75 & 3.45 \\
\hline & $\begin{array}{c}\text { Simpson } \\
\text { dominance } \\
\text { index }\end{array}$ & 0.03 & 0.05 & 0.05 & 0.05 & 0.04 & 0.04 & 0.04 & 0.03 & 0.03 & 0.04 & 0.03 & 0.05 & 0.03 & 0.05 \\
\hline & $\begin{array}{c}\text { Pileou's } \\
\text { evenness index }\end{array}$ & 0.95 & 0.86 & 0.87 & 0.89 & 0.9 & 0.91 & 0.89 & 0.93 & 0.94 & 0.91 & 0.94 & 0.88 & 0.92 & 0.86 \\
\hline
\end{tabular}




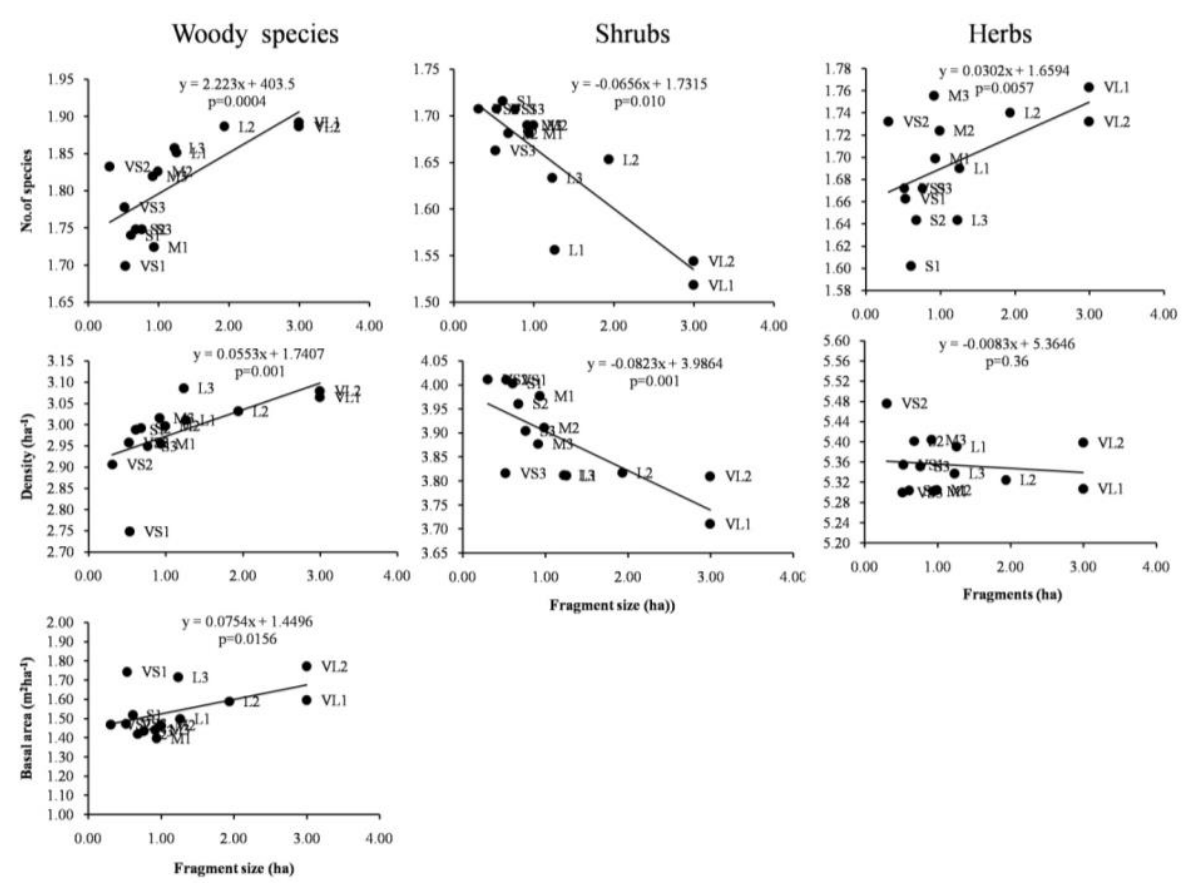

Figure 2. Vegetation characteristics along a fragment size gradient

\section{Forest floor litter}

The standing crop of litter on the forest floor showed an increasing trend along the fragment size gradient from $229 \mathrm{~g} \mathrm{~m}^{-2}$ in $\mathrm{S} 1$ to $371 \mathrm{~g} \mathrm{~m}^{-2}$ in L1 $\left(371 \mathrm{~g} \mathrm{~m}^{-2}\right)$ (Table 2). The proportional fraction of the various litter fractions also varied in the forest fragments and leaf litter mass ranged from 152 to $192 \mathrm{~g} \mathrm{~m}^{-2}$, while woody and miscellaneous fractions ranged from 61 to $95 \mathrm{~g} \mathrm{~m}^{-2}$ and 31 to $45 \mathrm{~g} \mathrm{~m}^{-2}$, respectively (Table 2). Thus, leaf litter (49-66\%) dominated the total litter accumulation across the fragments while the woody and miscellaneous fractions contributed to 21 to $30 \%$ and 12 to $16 \%$ of the total litter accumulation, respectively (Table 2). In terms of seasonality, the various fractions exhibited a more or less similar seasonal pattern with litter accumulation on the forest floor being maximum during winter (274-474 $\mathrm{g} \mathrm{m}^{-2}$ ) followed by spring (233-405 $\left.\mathrm{g} \mathrm{m}^{-2}\right)$, and rainy (203-342 $\left.\mathrm{g} \mathrm{m}^{-2}\right)$ and minimum during autumn $\left(179-265 \mathrm{~g} \mathrm{~m}^{-2}\right)$.

\section{Litterfall}

The total litterfall varied significantly $(\mathrm{p}<0.05)$ across the fragments and months and ranged from 266 to $608 \mathrm{~g} \mathrm{~m}^{-2}$ with the highest mean litterfall recorded in VL $\left(451.39 \mathrm{~g} \mathrm{~m}^{-2}\right)$ and $\mathrm{L}\left(418.70 \mathrm{~g} \mathrm{~m}^{-2}\right)$ fragments and lowest in VS $\left(316.64 \mathrm{~g} \mathrm{~m}^{-2}\right)$ fragment classes (Table 3, Figure 3). Leaf litter constituted about 40 to $57 \%$ of total litterfall in all the forest fragments, while woody and miscellaneous fraction, mainly composed of reproductive parts, contributed 26 to $41 \%$ and 13 to $23 \%$ of the total litterfall, respectively (Table 3). A proportional increase of leaf and miscellaneous litter along the increasing fragment size was observed, whereas woody litter decreased proportionally with increasing fragment size (Table 3, Figure 3). Litterfall production showed strong seasonal variability with maximum litterfall occurring during the cold and dry period (winter to spring) and minimum during autumn (Figure 4). The leaf litter showed similar seasonal patterns to the total litterfall. The woody litterfall occurred mainly from the late dry season 
(March-May) to the beginning of rainy season (May-June) while the miscellaneous fraction peaked between September and November. The highest monthly litterfall contributed $45.2 \%$ of the total annual litterfall and the lowest monthly contribution was only $1.3 \%$.

Table 2. Mean dry weight $\left(\mathrm{g} \mathrm{m}^{-2}\right)$ of different fractions of forest floor litter in the studied forest fragments

\begin{tabular}{|c|c|c|c|c|c|c|c|c|}
\hline \multirow{3}{*}{ Fragments } & \multicolumn{4}{|c|}{ First year } & \multicolumn{4}{|c|}{ Second year } \\
\hline & \multicolumn{8}{|c|}{ Litter Fractions } \\
\hline & Leaf & Woody & Miscellaneous & Total & Leaf & Woody & Miscellaneous & Total \\
\hline VS1 & $\begin{array}{l}187.80 \\
(58.02)\end{array}$ & $\begin{array}{c}84.37 \\
(26.07)\end{array}$ & $\begin{array}{c}51.52 \\
(15.92)\end{array}$ & 323.69 & $\begin{array}{l}183.81 \\
(58.96)\end{array}$ & $\begin{array}{l}78.56 \\
(25.20)\end{array}$ & $\begin{array}{c}49.41 \\
(15.85)\end{array}$ & 311.78 \\
\hline VS2 & $\begin{array}{l}149.61 \\
(62.08)\end{array}$ & $\begin{array}{c}52.49 \\
(21.78)\end{array}$ & $\begin{array}{l}38.88 \\
(16.13)\end{array}$ & 240.98 & $\begin{array}{l}148.52 \\
(63.50)\end{array}$ & $\begin{array}{l}48.59 \\
(20.77)\end{array}$ & $\begin{array}{c}36.79 \\
(15.73)\end{array}$ & 233.90 \\
\hline VS3 & $\begin{array}{l}180.63 \\
(60.43) \\
\end{array}$ & $\begin{array}{c}72.26 \\
(24.18) \\
\end{array}$ & $\begin{array}{r}46.00 \\
(15.39) \\
\end{array}$ & 298.89 & $\begin{array}{c}158.95 \\
(58.720 \\
\end{array}$ & $\begin{array}{c}62.97 \\
(23.26) \\
\end{array}$ & $\begin{array}{c}48.76 \\
(18.01) \\
\end{array}$ & 270.68 \\
\hline $\mathrm{S} 1$ & $\begin{array}{l}148.27 \\
(64.29)\end{array}$ & $\begin{array}{c}53.69 \\
(23.28)\end{array}$ & $\begin{array}{l}28.66 \\
(12.43)\end{array}$ & 230.62 & $\begin{array}{l}149.14 \\
(65.25)\end{array}$ & $\begin{array}{l}51.84 \\
(22.68)\end{array}$ & $\begin{array}{c}27.57 \\
(12.06)\end{array}$ & 228.55 \\
\hline S2 & $\begin{array}{l}164.56 \\
(59.92)\end{array}$ & $\begin{array}{c}76.97 \\
(28.03)\end{array}$ & $\begin{array}{c}33.09 \\
(12.05)\end{array}$ & 274.62 & $\begin{array}{l}155.26 \\
(60.77)\end{array}$ & $\begin{array}{l}67.69 \\
(26.50)\end{array}$ & $\begin{array}{c}32.53 \\
(12.73)\end{array}$ & 255.48 \\
\hline S3 & $\begin{array}{l}147.41 \\
(63.35) \\
\end{array}$ & $\begin{array}{c}55.53 \\
(23.86) \\
\end{array}$ & $\begin{array}{c}29.75 \\
(12.79) \\
\end{array}$ & 232.69 & $\begin{array}{l}145.67 \\
(61.51)\end{array}$ & $\begin{array}{c}59.23 \\
(25.01)\end{array}$ & $\begin{array}{c}31.94 \\
(13.49) \\
\end{array}$ & 236.84 \\
\hline M1 & $\begin{array}{l}158.17 \\
(63.39)\end{array}$ & $\begin{array}{c}57.09 \\
(22.88)\end{array}$ & $\begin{array}{c}34.27 \\
(13.73)\end{array}$ & 249.53 & $\begin{array}{l}152.43 \\
(65.67)\end{array}$ & $\begin{array}{l}51.16 \\
(22.04)\end{array}$ & $\begin{array}{c}28.54 \\
(12.29)\end{array}$ & 232.13 \\
\hline M2 & $\begin{array}{l}187.80 \\
(61.25)\end{array}$ & $\begin{array}{c}82.53 \\
(26.92)\end{array}$ & $\begin{array}{c}36.26 \\
(11.83)\end{array}$ & 306.59 & $\begin{array}{l}170.84 \\
(60.75)\end{array}$ & $\begin{array}{l}73.48 \\
(26.13)\end{array}$ & $\begin{array}{c}36.89 \\
(13.12)\end{array}$ & 281.21 \\
\hline M3 & $\begin{array}{l}159.06 \\
(60.76)\end{array}$ & $\begin{array}{c}67.32 \\
(25.71) \\
\end{array}$ & $\begin{array}{c}35.42 \\
(13.53) \\
\end{array}$ & 261.80 & $\begin{array}{r}153.90 \\
(59.90) \\
\end{array}$ & $\begin{array}{c}63.02 \\
(24.53) \\
\end{array}$ & $\begin{array}{c}40.00 \\
(15.57) \\
\end{array}$ & 256.92 \\
\hline L1 & $\begin{array}{l}182.97 \\
(49.27)\end{array}$ & $\begin{array}{c}99.29 \\
(26.74)\end{array}$ & $\begin{array}{l}89.07 \\
(23.99)\end{array}$ & 371.33 & $\begin{array}{l}160.80 \\
(59.94)\end{array}$ & $\begin{array}{c}75.98 \\
(28.32)\end{array}$ & $\begin{array}{l}31.48 \\
(11.73)\end{array}$ & 268.26 \\
\hline L2 & $\begin{array}{l}168.40 \\
(61.04)\end{array}$ & $\begin{array}{l}74.41 \\
(26.97)\end{array}$ & $\begin{array}{c}33.09 \\
(11.99)\end{array}$ & 275.90 & $\begin{array}{l}159.23 \\
(62.96)\end{array}$ & $\begin{array}{l}63.68 \\
(25.18)\end{array}$ & $\begin{array}{l}30.00 \\
(11.86)\end{array}$ & 252.91 \\
\hline L3 & $\begin{array}{l}187.80 \\
(57.74) \\
\end{array}$ & $\begin{array}{r}99.29 \\
(30.53) \\
\end{array}$ & $\begin{array}{c}38.15 \\
(11.73) \\
\end{array}$ & 325.24 & $\begin{array}{l}179.95 \\
(63.15) \\
\end{array}$ & $\begin{array}{l}68.75 \\
(24.13) \\
\end{array}$ & $\begin{array}{c}36.24 \\
(12.72) \\
\end{array}$ & 284.94 \\
\hline VL1 & $\begin{array}{l}180.76 \\
(59.27)\end{array}$ & $\begin{array}{c}85.41 \\
(28.01)\end{array}$ & $\begin{array}{c}38.79 \\
(12.72)\end{array}$ & 304.96 & $\begin{array}{l}186.63 \\
(57.45)\end{array}$ & $\begin{array}{c}98.19 \\
(30.23)\end{array}$ & $\begin{array}{c}40.01 \\
(12.32)\end{array}$ & 324.83 \\
\hline VL2 & $\begin{array}{r}199.69 \\
(59.87) \\
\end{array}$ & $\begin{array}{c}91.26 \\
(27.36)\end{array}$ & $\begin{array}{r}42.58 \\
(12.77)\end{array}$ & 333.53 & $\begin{array}{l}202.37 \\
(57.55)\end{array}$ & $\begin{array}{r}105.38 \\
(29.97)\end{array}$ & $\begin{array}{c}43.90 \\
(12.48)\end{array}$ & 351.65 \\
\hline
\end{tabular}

Values in parentheses are percentages of total

\section{Nutrient return through litterfall}

Potential nutrient $(\mathrm{N}$ and $\mathrm{P})$ return through the different litter fractions varied significantly $(\mathrm{p}<0.05)$ with the highest input through leaf $\left(17.64\right.$ to $29.21 \mathrm{~kg} \mathrm{ha}^{-1}$ for $\mathrm{N}$ and 0.86 to $1.31 \mathrm{~kg} \mathrm{ha}^{-1}$ for $\mathrm{P}$ ) followed by woody (7.10 to $16.38 \mathrm{~kg} \mathrm{ha}^{-1} \mathrm{~N}$ and 0.39 to $0.77 \mathrm{~kg} \mathrm{ha}^{-1} \mathrm{P}$ ) and miscellaneous (3.94 to $8.65 \mathrm{~kg} \mathrm{ha}^{-1} \mathrm{~N}$ and 0.18 to $0.32 \mathrm{~kg} \mathrm{ha}^{-1} \mathrm{P}$ ) litter (Table 4). The highest potential $\mathrm{N}$ return occurred through leaf litter $(55 \%$ for $\mathrm{M}$ to $61 \%$ of the total for VS) followed by the woody fractions that had higher $\mathrm{N}$ returns in $\mathrm{M}, \mathrm{L}$ and VL. The potential $\mathrm{P}$ return from the different fraction of litterfall in the studied fragments followed a pattern similar to $\mathrm{N}$ return with maximum input from the leaf (55-59\%), followed by the woody (28-32\%) and miscellaneous (13-14\%) fractions. Although, leaf litter fraction had the highest input of $\mathrm{N}$ and $\mathrm{P}$ across the forests fragments, however, the comparative contributory nutrient return through leaf litter to the total nutrient return exhibited a declining trend along the fragment size gradient, whereas nutrient return through woody litter showed an upward increase (Table 4). 
Table 3. Annual litter fall $\left(\mathrm{g} \mathrm{m}^{-2}\right)$ in the studied forest fragments

\begin{tabular}{|c|c|c|c|c|c|c|c|c|}
\hline \multirow{3}{*}{ Fragments } & \multicolumn{4}{|c|}{ First year } & \multicolumn{4}{|c|}{ Second year } \\
\hline & \multicolumn{8}{|c|}{ Litter Fractions } \\
\hline & Leaf & Woody & Miscellaneous & Total & Leaf & Woody & Miscellaneous & Total \\
\hline VS1 & $\begin{array}{l}169.00 \\
(56.51)\end{array}$ & $\begin{array}{c}90.30 \\
(30.19)\end{array}$ & $\begin{array}{c}39.76 \\
(13.30)\end{array}$ & 299.06 & $\begin{array}{l}170.89 \\
(45.45)\end{array}$ & $\begin{array}{l}155.98 \\
(41.48)\end{array}$ & $\begin{array}{c}49.16 \\
(13.07)\end{array}$ & 376.02 \\
\hline VS2 & $\begin{array}{l}150.80 \\
(56.76)\end{array}$ & $\begin{array}{c}69.61 \\
(26.20)\end{array}$ & $\begin{array}{c}45.27 \\
(17.04)\end{array}$ & 265.68 & $\begin{array}{l}158.34 \\
(48.06)\end{array}$ & $\begin{array}{l}124.08 \\
(37.66)\end{array}$ & $\begin{array}{c}47.02 \\
(14.27)\end{array}$ & 329.44 \\
\hline VS3 & $\begin{array}{l}150.34 \\
(46.94) \\
\end{array}$ & $\begin{array}{l}117.71 \\
(36.76) \\
\end{array}$ & $\begin{array}{c}52.20 \\
(16.30) \\
\end{array}$ & 320.25 & $\begin{array}{l}150.36 \\
(48.60) \\
\end{array}$ & $\begin{array}{l}111.84 \\
(36.15) \\
\end{array}$ & $\begin{array}{c}47.19 \\
(15.25) \\
\end{array}$ & 309.39 \\
\hline $\mathrm{S} 1$ & $\begin{array}{l}170.56 \\
(44.51)\end{array}$ & $\begin{array}{l}155.19 \\
(40.50)\end{array}$ & $\begin{array}{c}57.46 \\
(15.00)\end{array}$ & 383.21 & $\begin{array}{l}146.08 \\
(39.83)\end{array}$ & $\begin{array}{l}137.17 \\
(37.40)\end{array}$ & $\begin{array}{l}83.50 \\
(22.77)\end{array}$ & 366.76 \\
\hline S2 & $\begin{array}{l}211.19 \\
(49.91)\end{array}$ & $\begin{array}{l}150.8 \\
(35.63)\end{array}$ & $\begin{array}{c}61.19 \\
(14.46)\end{array}$ & 423.19 & $\begin{array}{l}204.95 \\
(46.26)\end{array}$ & $\begin{array}{l}171.51 \\
(38.71)\end{array}$ & $\begin{array}{c}66.59 \\
(15.03)\end{array}$ & 443.06 \\
\hline S3 & $\begin{array}{c}157.3 \\
(47.70) \\
\end{array}$ & $\begin{array}{r}123.39 \\
(37.42) \\
\end{array}$ & $\begin{array}{c}49.05 \\
(14.880 \\
\end{array}$ & 329.75 & $\begin{array}{l}154.21 \\
(40.55)\end{array}$ & $\begin{array}{l}149.16 \\
(39.22) \\
\end{array}$ & $\begin{array}{l}76.96 \\
(20.24) \\
\end{array}$ & 380.32 \\
\hline M1 & $\begin{array}{l}177.36 \\
(47.06)\end{array}$ & $\begin{array}{c}139.3 \\
(36.96)\end{array}$ & $\begin{array}{c}60.19 \\
(15.97)\end{array}$ & 376.86 & $\begin{array}{l}148.26 \\
(44.52)\end{array}$ & $\begin{array}{l}129.50 \\
(38.89)\end{array}$ & $\begin{array}{c}55.24 \\
(16.59)\end{array}$ & 332.99 \\
\hline M2 & $\begin{array}{l}188.07 \\
(53.16)\end{array}$ & $\begin{array}{l}112.83 \\
(31.89)\end{array}$ & $\begin{array}{c}52.91 \\
(14.95)\end{array}$ & 353.81 & $\begin{array}{l}138.82 \\
(51.54)\end{array}$ & $\begin{array}{c}94.03 \\
(34.91)\end{array}$ & $\begin{array}{c}36.50 \\
(13.55)\end{array}$ & 269.34 \\
\hline M3 & $\begin{array}{l}190.04 \\
(49.45) \\
\end{array}$ & $\begin{array}{l}137.60 \\
(35.81) \\
\end{array}$ & $\begin{array}{r}56.66 \\
(14.74) \\
\end{array}$ & 384.31 & $\begin{array}{l}226.72 \\
(47.87) \\
\end{array}$ & $\begin{array}{r}169.83 \\
(35.86) \\
\end{array}$ & $\begin{array}{r}77.04 \\
(16.27) \\
\end{array}$ & 473.59 \\
\hline L1 & $\begin{array}{l}149.31 \\
(51.75)\end{array}$ & $\begin{array}{c}93.38 \\
(32.37)\end{array}$ & $\begin{array}{c}45.82 \\
(15.88)\end{array}$ & 288.51 & $\begin{array}{l}170.95 \\
(52.73)\end{array}$ & $\begin{array}{c}83.60 \\
(25.79)\end{array}$ & $\begin{array}{c}69.63 \\
(21.48)\end{array}$ & 324.18 \\
\hline $\mathrm{L} 2$ & $\begin{array}{l}190.04 \\
(49.45)\end{array}$ & $\begin{array}{l}137.6 \\
(35.81)\end{array}$ & $\begin{array}{l}56.66 \\
(14.74)\end{array}$ & 384.31 & $\begin{array}{l}188.07 \\
(53.16)\end{array}$ & $\begin{array}{l}112.83 \\
(31.89)\end{array}$ & $\begin{array}{c}52.91 \\
(14.95)\end{array}$ & 353.81 \\
\hline L3 & $\begin{array}{l}274.51 \\
(49.59) \\
\end{array}$ & $\begin{array}{l}193.51 \\
(34.96)\end{array}$ & $\begin{array}{c}85.56 \\
(15.46)\end{array}$ & 553.59 & $\begin{array}{l}308.18 \\
(50.70)\end{array}$ & $\begin{array}{l}212.32 \\
(34.93)\end{array}$ & $\begin{array}{c}87.32 \\
(14.37)\end{array}$ & 607.82 \\
\hline VL1 & $\begin{array}{l}202.83 \\
(48.49)\end{array}$ & $\begin{array}{l}119.73 \\
(28.62)\end{array}$ & $\begin{array}{c}95.77 \\
(22.89)\end{array}$ & 418.33 & $\begin{array}{l}236.65 \\
(49.86)\end{array}$ & $\begin{array}{l}140.38 \\
(29.58)\end{array}$ & $\begin{array}{c}97.61 \\
(20.56)\end{array}$ & 474.65 \\
\hline VL2 & $\begin{array}{l}224.48 \\
(54.05)\end{array}$ & $\begin{array}{l}127.73 \\
(30.75)\end{array}$ & $\begin{array}{c}63.13 \\
(15.20) \\
\end{array}$ & 415.34 & $\begin{array}{l}247.78 \\
(49.83)\end{array}$ & $\begin{array}{l}159.47 \\
(32.07)\end{array}$ & $\begin{array}{c}89.98 \\
(18.10)\end{array}$ & 497.23 \\
\hline
\end{tabular}

Values in parentheses are percentages of total
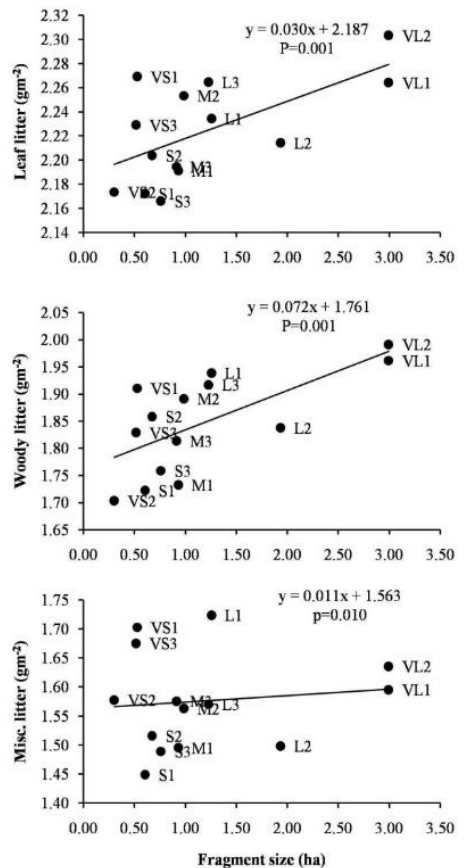
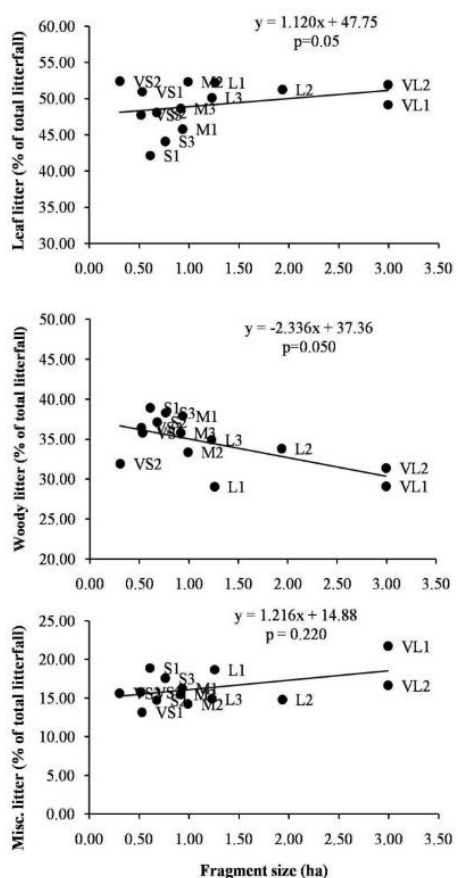

Figure 3. Effect of fragment size on litterfall and the percentage variation of the litter fractions (\% of total litterfall) along a fragment size gradient 

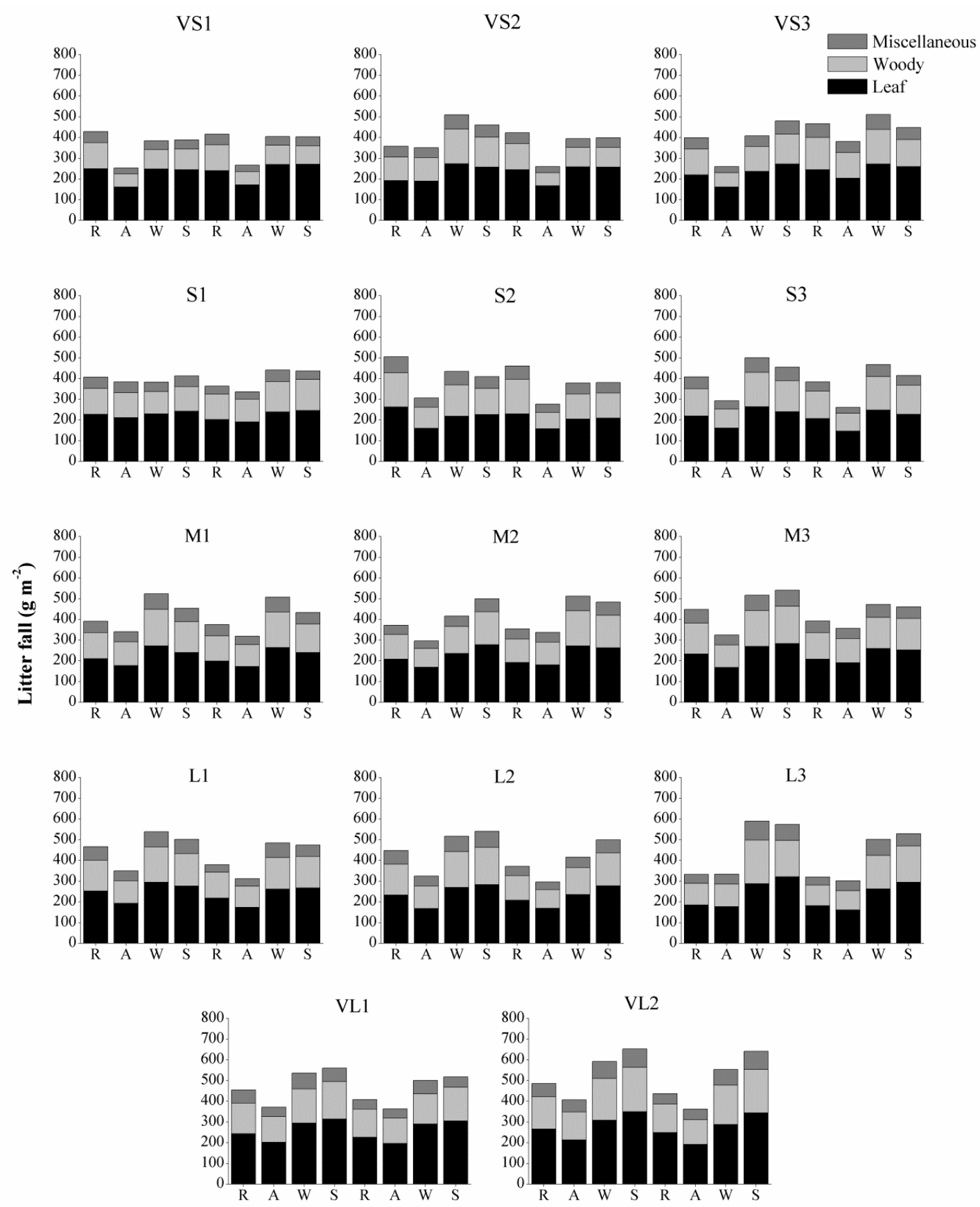

Seasons

Figure 4. Seasonal variation in litterfall $\left(\mathrm{g} \mathrm{m}^{-2}\right)$ across the various forest fragments ( $R$ : Rainy; A: Autumn; W: Winter; S: Spring)

\section{Relationship of litter with vegetation characteristics}

The relationship of litterfall dynamics with vegetation characteristics showed that leaf and woody litter fractions of litterfall exhibited positive correlation with stand density and basal area of woody species and were proportionally higher in fragments with high density and basal area (Table 5) thus explaining the comparable litterfall values of VS3, S1, S2, S3, M1, M2 and L1 (Table 5). 
Table 4. Annual input $\left(\mathrm{kg} \mathrm{ha}^{-1}\right)$ of $N$ and $P$ through different fractions of litter in the studied forest fragments

\begin{tabular}{|c|c|c|c|c|c|c|c|c|}
\hline \multirow{2}{*}{ Fragments } & \multicolumn{4}{|c|}{ Nitrogen } & \multicolumn{4}{|c|}{ Phosphorus } \\
\hline & Leaf & Woody & Miscellaneous & Total & Leaf & Woody & Miscellaneous & Total \\
\hline VS1 & $\begin{array}{c}21.55 \\
(65.51)\end{array}$ & $\begin{array}{c}7.35 \\
(22.34)\end{array}$ & $\begin{array}{c}4.00 \\
(12.14)\end{array}$ & 32.90 & $\begin{array}{c}1.06 \\
(63.94)\end{array}$ & $\begin{array}{c}0.41 \\
(24.55)\end{array}$ & $\begin{array}{c}0.19 \\
(11.52)\end{array}$ & 1.65 \\
\hline VS2 & $\begin{array}{c}20.54 \\
(59.76)\end{array}$ & $\begin{array}{c}8.74 \\
(25.43)\end{array}$ & $\begin{array}{c}5.09 \\
(14.81)\end{array}$ & 34.37 & $\begin{array}{c}0.99 \\
(58.46)\end{array}$ & $\begin{array}{c}0.48 \\
(28.49)\end{array}$ & $\begin{array}{c}0.22 \\
(13.06)\end{array}$ & 1.69 \\
\hline VS3 & $\begin{array}{c}20.87 \\
(56.74) \\
\end{array}$ & $\begin{array}{c}10.24 \\
(27.84)\end{array}$ & $\begin{array}{c}5.68 \\
(15.43)\end{array}$ & 36.79 & $\begin{array}{c}0.99 \\
(55.34)\end{array}$ & $\begin{array}{c}0.55 \\
(30.62)\end{array}$ & $\begin{array}{c}0.25 \\
(14.04)\end{array}$ & 1.78 \\
\hline S1 & $\begin{array}{c}20.45 \\
(60.37)\end{array}$ & $\begin{array}{c}8.65 \\
(25.52)\end{array}$ & $\begin{array}{c}4.78 \\
(14.11)\end{array}$ & 33.88 & $\begin{array}{c}0.98 \\
(56.03)\end{array}$ & $\begin{array}{c}0.56 \\
(32.18)\end{array}$ & $\begin{array}{c}0.21 \\
(11.78)\end{array}$ & 1.74 \\
\hline S2 & $\begin{array}{c}18.40 \\
(56.99)\end{array}$ & $\begin{array}{c}8.69 \\
(26.92)\end{array}$ & $\begin{array}{c}5.20 \\
(16.09)\end{array}$ & 32.29 & $\begin{array}{c}0.90 \\
(54.22)\end{array}$ & $\begin{array}{c}0.52 \\
(31.33)\end{array}$ & $\begin{array}{c}0.24 \\
(14.46)\end{array}$ & 1.66 \\
\hline S3 & $\begin{array}{c}19.23 \\
(57.21)\end{array}$ & $\begin{array}{c}9.26 \\
(27.54) \\
\end{array}$ & $\begin{array}{c}5.13 \\
(15.25) \\
\end{array}$ & 33.61 & $\begin{array}{c}0.91 \\
(54.49) \\
\end{array}$ & $\begin{array}{c}0.54 \\
(32.04) \\
\end{array}$ & $\begin{array}{c}0.23 \\
(13.47) \\
\end{array}$ & 1.67 \\
\hline M1 & $\begin{array}{c}20.29 \\
(55.41)\end{array}$ & $\begin{array}{l}10.16 \\
(27.75)\end{array}$ & $\begin{array}{c}6.17 \\
(16.84)\end{array}$ & 36.61 & $\begin{array}{c}0.99 \\
(55.00)\end{array}$ & $\begin{array}{c}0.56 \\
(31.11)\end{array}$ & $\begin{array}{c}0.25 \\
(13.89)\end{array}$ & 1.80 \\
\hline M2 & $\begin{array}{l}20.31 \\
(56.45)\end{array}$ & $\begin{array}{l}10.56 \\
(29.34)\end{array}$ & $\begin{array}{c}5.11 \\
(14.21)\end{array}$ & 35.97 & $\begin{array}{c}1.01 \\
(57.59)\end{array}$ & $\begin{array}{c}0.52 \\
(29.80)\end{array}$ & $\begin{array}{c}0.22 \\
(12.61)\end{array}$ & 1.75 \\
\hline M3 & $\begin{array}{c}21.02 \\
(53.94) \\
\end{array}$ & $\begin{array}{l}11.82 \\
(30.34)\end{array}$ & $\begin{array}{c}6.13 \\
(15.72)\end{array}$ & 38.96 & $\begin{array}{c}1.07 \\
(55.76) \\
\end{array}$ & $\begin{array}{c}0.59 \\
(30.63) \\
\end{array}$ & $\begin{array}{c}0.26 \\
(13.61) \\
\end{array}$ & 1.91 \\
\hline L1 & $\begin{array}{c}22.75 \\
(57.57)\end{array}$ & $\begin{array}{c}10.77 \\
(27.25)\end{array}$ & $\begin{array}{c}6.00 \\
(15.19)\end{array}$ & 39.51 & $\begin{array}{c}1.06 \\
(56.99)\end{array}$ & $\begin{array}{c}0.56 \\
(29.84)\end{array}$ & $\begin{array}{c}0.25 \\
(13.17)\end{array}$ & 1.86 \\
\hline L2 & $\begin{array}{c}21.71 \\
(56.53)\end{array}$ & $\begin{array}{l}11.05 \\
(28.77)\end{array}$ & $\begin{array}{c}5.65 \\
(14.70)\end{array}$ & 38.40 & $\begin{array}{c}1.01 \\
(55.83)\end{array}$ & $\begin{array}{c}0.56 \\
(30.83)\end{array}$ & $\begin{array}{c}0.24 \\
(13.33)\end{array}$ & 1.80 \\
\hline L3 & $\begin{array}{c}22.79 \\
(55.34)\end{array}$ & $\begin{array}{l}11.44 \\
(27.77)\end{array}$ & $\begin{array}{c}6.96 \\
(16.89)\end{array}$ & 41.18 & $\begin{array}{c}1.03 \\
(55.38)\end{array}$ & $\begin{array}{c}0.56 \\
(29.84)\end{array}$ & $\begin{array}{c}0.28 \\
(14.78)\end{array}$ & 1.86 \\
\hline VL1 & $\begin{array}{c}26.13 \\
(58.28)\end{array}$ & $\begin{array}{l}12.21 \\
(27.23)\end{array}$ & $\begin{array}{c}6.50 \\
(14.49)\end{array}$ & 44.84 & $\begin{array}{c}1.17 \\
(56.66)\end{array}$ & $\begin{array}{c}0.64 \\
(30.99)\end{array}$ & $\begin{array}{c}0.26 \\
(12.35)\end{array}$ & 2.07 \\
\hline VL2 & $\begin{array}{c}28.17 \\
(56.31) \\
\end{array}$ & $\begin{array}{c}14.26 \\
(28.51) \\
\end{array}$ & $\begin{array}{c}7.59 \\
(15.18) \\
\end{array}$ & 50.02 & $\begin{array}{c}1.25 \\
(54.59) \\
\end{array}$ & $\begin{array}{c}0.74 \\
(32.10)\end{array}$ & $\begin{array}{c}0.31 \\
(13.32)\end{array}$ & 2.29 \\
\hline
\end{tabular}

Values in parentheses are percentages of total

Table 5. Relationship of vegetation characteristics and litterfall with fragment size (ha) and the influence of density (individuals $\left.\mathrm{ha}^{-1}\right)$ and basal area $\left(\mathrm{m}^{2} \mathrm{ha}^{-1}\right)$ of woody individuals on litterfall dynamics in the studied forest fragments

\begin{tabular}{c|c|c|c}
\hline Parameters & Regression equation & r & P \\
\hline Relationship with fragment size & & & \\
Woody species richness & $\mathrm{y}=2.223 \mathrm{x}+403.5$ & 0.726 & 0.0004 \\
Woody stand density & $\mathrm{y}=0.055 \mathrm{x}+1.740$ & 0.619 & $<0.001$ \\
Woody basal area & $\mathrm{y}=0.075 \mathrm{x}+1.449$ & 0.525 & 0.016 \\
Shrub species richness & $\mathrm{y}=-0.066 \mathrm{x}+1.731$ & -0.863 & 0.011 \\
Shrub stand density & $\mathrm{y}=-0.082 \mathrm{x}+3.986$ & -0.752 & $<0.001$ \\
Herbaceous species richness & $\mathrm{y}=0.030 \mathrm{x}+1.659$ & 0.528 & 0.006 \\
Herbaceous stand density & $\mathrm{y}=-0.008 \mathrm{x}+5.365$ & -0.121 & $<0.001$ \\
Leaf litterfall & $\mathrm{y}=0.030 \mathrm{x}+2.187$ & 0.607 & $<0.001$ \\
Woody litterfall & $\mathrm{y}=0.072 \mathrm{x}+1.761$ & 0.669 & $<0.001$ \\
Miscellaneous litterfall & $\mathrm{y}=0.011 \mathrm{x}+1.563$ & 0.126 & 0.01084 \\
\hline Relationship with density & & & \\
Leaf litterfall & $\mathrm{y}=0.605 \mathrm{x}+0.456$ & 0.170 & 0.574 \\
Woody litterfall & $\mathrm{y}=0.383 \mathrm{x}+0.968$ & 0.322 & 0.224 \\
Miscellaneous litterfall & $\mathrm{y}=0.869 \mathrm{x}-0.808$ & -0.226 & 0.0161 \\
\hline Relationship with basal area & & & \\
Leaf litterfall & $\mathrm{y}=0.401 \mathrm{x}+1.647$ & 0.784 & 0.0482 \\
Woody litterfall & $\mathrm{y}=0.208 \mathrm{x}+1.793$ & 0.656 & 0.187 \\
Miscellaneous litterfall & $\mathrm{y}=0.255 \mathrm{x}+1.393$ & 0.386 & 0.896 \\
\hline
\end{tabular}




\section{Discussion}

The effect of fragmentation on plant diversity was realized by a structural and compositional change in floristic diversity, species richness as well as density of woody species along a fragment size gradient. The high species richness, and density of woody species in larger fragments was attributed to low disturbances, habitat suitability and favorable growth conditions (Mir and Upadhaya, 2017) whereas, low species richness, basal area and tree density in very small and small fragment categories may be attributed to repeated human disturbances such as small timber and NTFPs extraction. Extraction of timber has been known to be a contributing factor to fragment degradation in many tropical and subtropical forest (Ochoa-Gaono et al., 2004; Cayuela et al., 2006; Echeverría et al., 2007; Santos et al., 2007; Lin and Cao, 2009; Majumdar et al., 2014; Pao and Upadhaya, 2017).

Disturbance provides high light accessibility that plays a key factor for the understory plant species to flourish (Tuomisto et al., 2003) in forest gaps (Sonnier et al., 2014). This was proven to be true in the present study as species richness of shrubs in the fragments showed a reverse trend to that of woody species with high species richness and density in the smaller fragments as compared to larger ones due to canopy openness, adequate light and favorable environmental conditions (Laurance and Vasconcelos, 2009). The species richness of herbs however, increased along the fragments size gradient, exhibiting similarity to woody species richness and corroborating the findings that fragments with larger core areas favour herbaceous species in comparison to shrubs, suggesting the importance of maintaining large core areas to conserve herbaceous forest specialist (Hofmeister et al., 2013).

The range (50 to 78) of woody species ( $\geq 5 \mathrm{~cm} \mathrm{dbh}$ ) richness recorded in the present study was higher than tropical dry forest of Puerto Rico (30 to 35 species, Murphy and Lugo, 1986), moist deciduous forest of Western Ghats (24-26 species, Valappil and Swarupanandan, 1996) and fragmented moist deciduous forest of West Tripura (31 to 61 species in eleven sites, Majumdar et al., 2014). Shannon-Wiener's diversity index (H') for woody species in the present study (3.09 to 3.72) was within the range for Indian forests (0.83 to 4.1, Parthasarathy et al., 1992).

The low density of woody species in smaller fragments could be attributed to disturbance. The stand density of woody species $(\geq 5 \mathrm{~cm} \mathrm{dbh})$ recorded in the present study (560 to 1220 individuals $\mathrm{ha}^{-1}$ ) is within the reported estimates from subtropical forests of Khasi Hills (1109 individuals ha $^{-1}$, Upadhaya, 2015) of Meghalaya, tropical rainforest forests of Congo Basin (144 to 868 individuals ha $^{-1}$, Ifo et al., 2016), evergreen forests of Thailand (602 to 992 individuals ha $^{-1}$, Lamotte et al., 1998) as well as fragmented moist deciduous forest ecosystems of northeast India (428 to $884 \mathrm{ha}^{-1}$, Majumdar et al., 2014).

The high basal area in larger fragments and in VS1 was due to the presence of bigger trees, while the reduction of basal area in smaller fragments was due to the removal of bigger trees. Such an increase in basal area with increasing fragment size has also been observed from other subtropical forest of the region (Tripathi et al., 2010; Pao and Upadhaya, 2017) and tropical rainforest of Western Ghats (Bhat et al., 2000). The stand basal area recorded in the present study (25.00 to $\left.59.3 \mathrm{~m}^{2} \mathrm{ha}^{1}\right)$ is close to the reported estimates from other subtropical forests of Jaintia Hills (36.52-77.44 $\mathrm{m}^{2} \mathrm{ha}^{-1}$, Upadhaya et al., 2003), and Khasi hills (30 to $38 \mathrm{~m}^{2} \mathrm{ha}^{-1}$, Upadhaya, 2015) in Meghalaya, Ishigaki and Okinawa Island Japan (42 to $43 \mathrm{~m}^{2} \mathrm{ha}^{-1}$, Feroz et al., 2015) and tropical forests of Myanmar (22.93-47.83 $\mathrm{m}^{2} \mathrm{ha}^{-1}$, Aye et al., 2014). The differences in the basal area of 
woody species ( $\geq 5 \mathrm{~cm} \mathrm{dbh}$ ) among the forest fragments may be attributed to the differences in species composition, the age of trees, the extent of disturbances and successional stages of the stands (Naidu and Kumar, 2016).

The intensity of anthropogenic disturbances through extraction and edge creation greatly influenced species dominance in the studied fragmented forests that may have future repercussions on the structure and composition, succession phases, and species restoration of these forests. Fragmentation may establish several transitional community associations which are mostly dominated by few local adaptable species through direct or indirect competitions as observed in the present study wherein the larger patches were characterized by nemoral species. The smaller patches were dominated by shrubs indicating the susceptibility of smaller fragments to external disturbances (Pao and Upadhaya, 2017). They were also prone to a larger number of gaps that would eventually affect tree regeneration and recruitment (Lovejoy et al., 1986; Turner and Corlett, 1996; Laurance et al., 1998).

The litter accumulation on the forest floor showed an increase along the fragment size gradient ( $229 \mathrm{~g} \mathrm{~m}^{-2}$ in $\mathrm{S} 1$ to $371 \mathrm{~g} \mathrm{~m}^{-2}$ in L1) and was within the reported estimates (217 to $2255 \mathrm{~g} \mathrm{~m}^{-2}$ ) for tropical-broadleaf semideciduous, -broadleaf deciduous and -broadleaf evergreen forests worldwide (Vogt et al., 1986). The values of forest floor litter were close to the reported estimates of tropical rainforests $\left(550 \mathrm{~g} \mathrm{~m}^{-2}\right.$, Morellato, 1992) but lower than the litter standing crop in a secondary lowland rain forest in Nigeria (830 to $940 \mathrm{~g} \mathrm{~m}^{-2}$, Odiwe and Muoghalu, 2003), sub-humid tropical Nigeria $\left(690-1170 \mathrm{~g} \mathrm{~m}^{-2}\right.$, Swift et al., 1981) and three tropical Australian rain forests $\left(250-1050 \mathrm{~g} \mathrm{~m}^{-2}\right.$, Spain, 1984).

Litterfall rates vary to a great extent among different forest fragments. The annual litterfall obtained in the present study $\left(266-608 \mathrm{~g} \mathrm{~m}^{-2} \mathrm{yr}^{-1}\right)$ were within the range (300-1100 $\mathrm{g} \mathrm{m}^{-2} \mathrm{yr}^{-1}$ ) recorded from different forest types (Vogt et al., 1986; Cuevas and Lugo, 1998; Lian and Zhang, 1998; Sundarapandian and Swamy, 1999; Alhamd and Hagihara, 2004; Zhang et al., 2014; Zhou et al., 2016). However, the values were lower than those recorded from tropical forests $\left(550-1200 \mathrm{~g} \mathrm{~m}^{-2} \mathrm{yr}^{-1}\right.$; Vitousek and Sanford, 1986), montane rain forests (700-760 $\mathrm{g} \mathrm{m}^{-2} \mathrm{yr}^{-1}$; Edwards, 1982; Vitousek et al., 1995) and tropical lowland forests (750 to $1530 \mathrm{~g} \mathrm{~m}^{-2} \mathrm{yr}^{-1}$; Bray and Gorham, 1964; Vitousek, 1984; Proctor, 1987). The high litter fall in VS1 and VS3 can be attributed to the comparable stand density and basal area to L3 and medium (M) fragments respectively, thus, suggesting litter production in forest ecosystems as a function of species composition, stand structure, age, quality as well as climatic factors (Haase, 1999; Sundarapandian and Swamy, 1999; Norgrove and Hauser, 2000; Yang et al., 2004). The variation in litter production among stands within the same climate range has been mainly attributed to species composition (Sundarapandian and Swamy, 1999) and an increase in litter production may also be explained by the competitive production principle (Kelty, 2006) according to which an admixture of species that have substantially different characteristics such as foliar phenology, shade tolerance, crown structure and root depth and phenology may use site resources more efficiently in producing materials, resulting in greater biomass than as they would in monocultures of the component species (Binkley et al., 1992). The high spatial heterogeneity in litterfall in fragments of $>80$ ha could be attributed to the variation in the biotic and abiotic conditions within each fragment as smaller fragments were less heterogeneous, likely due to edge effects and microclimatic conditions (such as wind and temperature) when compared with larger fragments (Laurance, 2004). 
The contribution of leaf litter (40 to 57\%) to the total litterfall in the present study ranges within the recorded estimates of leaf litter fraction for most subtropical evergreen forests (50 to 80\%, Tu et al., 1993; Lian and Zhang, 1998; Lin et al., 1999) as well as tropical forests in China (56 to 61\%, Tang et al., 2010) and subtropical broad-leaved forests in Japan (52.6 to 58.4\%, Alhamd and Hagihara, 2004). The relatively higher proportion of leaves in the litter fall has been extensively reported from many forest ecosystems (Figueiredo-Filho et al., 2003; Pinto et al., 2008) and is attributed to the fact that, in addition to being the physiologically active organs responsible for photosynthesis (Epstein and Bloom, 2006), leaves are also responsible for the transfer of carbon and nutrients to forest soils, thus causative, to maintain and even enrich their fertility.

The seasonal patterns of leaf, woody and miscellaneous fractions of litterfall showed unanimity to the total litterfall with peak observed during March and minimum values recorded during September that was in conjunction with reports from subtropical evergreen forest of China wherein old leaves of most species were generally replaced by new leaves in spring as well as tropical forests where litter peaks occurred mostly in spring or winter, corresponding to the drought season (Zhang et al., 2014). This seasonal attribute of peaks observed in litterfall in spring, summer and autumn have been measured in tropical climates (Singh et al., 1989; Sundarapandian and Swamy, 1999) and may be associated with physiological leaf senescence (Liang, 1994; Lin et al., 1999; Yang et al., 2004).

Mean annual potential returns of $\mathrm{N}$ through litterfall in the studied fragments (32.3-50.02 kg ha-1) were close to the reported estimates from subtropical broadleaved forests (36-128 $\mathrm{kg} \mathrm{ha}^{-1}$, Deng et al., 1993; Weng et al., 1993; Zheng et al., 1995; Lin et al., 1999; Yang et al., 2004) as well as pure Chinese fir plantations in Tianlin (39 kg ha-1, Liang, 1994) and Huitong (37 kg ha-1, Tian and Zhao, 1989) in China. The potential returns of $\mathrm{P}$ through litterfall $\left(1.65-2.29 \mathrm{~kg} \mathrm{ha}^{-1}\right)$ were also close to the reported estimates from subtropical rain forest in Hexi (3.8 $\mathrm{kg} \mathrm{ha}^{-1}$, Zheng et al., 1995) in China as well as a primary Lithocarpus xylocarpus forest in Ailao mountain $\left(1.7 \mathrm{~kg} \mathrm{ha}^{-1}\right.$, Deng et al., 1993) and an old-growth evergreen broadleaved forest in Dinghu mountain $\left(5.9 \mathrm{~kg} \mathrm{ha}^{-1}\right.$, Weng et al., 1993). However, the values were lower than the range $\left(2.4-6.6 \mathrm{~kg} \mathrm{ha}^{-1}\right.$, Yang et al., 2004) reported from four plantations compared with a natural subtropical forest in Sanming, Fujian, China. N and P are the major limiting nutrients for tree growth in many subtropical forests because of high soil acidity; hence the relatively high return of $\mathrm{N}$ and $\mathrm{P}$ through litterfall makes the broadleaved species more advantageous over conifers in nutrient supply, especially in the surface soil horizons (Yang et al., 1993)

\section{Conclusion}

Understanding the patterns of species occurrence and ecosystem processes has proven to be challenging because of the idiosyncratic responses of species to habitat fragmentation. Forest fragmentation reduces the ability of the remnant forests to maintain their original biodiversity and ecological processes (Echeverría et al., 2007). In the present study, the variation in species composition in remnant fragments can be attributed to the interaction of fragmentation and human disturbances such as timber and fuel wood extraction as well as NTFP collection (Debinski and Holt, 2000; Cochrane and Laurance, 2002; Santos et al., 2007; Pao and Upadhaya, 2017). The proximity to human settlements has aided the extraction of species for fuel wood and timbers resulting in thinning of forests and fragmentation of the patches (Kumar et al., 2008; Upadhaya et al., 2014) and 
thus impose a serious threat to the biodiversity of the area. The presence of nemoral species in the larger patches and, smaller patches being characterized by shrubs species indicated the susceptibility of smaller fragments to external disturbances (Pao and Upadhaya, 2017). Nutrients are often limiting resources, and so facilitated nutrient cycling of dead organic material is essential for continued forest productivity. However, nutrient cycling studies should also consider other vital aspects such as decomposition rate of litters, microbial mediated processes involved in the conversion of organic nutrients into available forms (Pao and Upadhaya, 2019) and its subsequent uptake through roots in detailed to understand the overall effect of fragmentation on structure and function of subtropical broadleaved forest ecosystems. Nevertheless, the findings of the present study demonstrated that fragmentation of forests mediate an urgent need for conservation and restoration measures to improve landscape connectivity, which will reduce extinction rates and help maintain ecosystem- functions and -services.

Funding. The authors are thankful to the Ministry of Environment, Forest and Climate Change (MoEF \& CC), Government of India for the financial support in the form of a research project (No. 14/25/ 2011ERS/RE).

Author contribution. KU conceived the idea, KU and KS designed the experimental layout, HSAB, AHM and GC carried out field and laboratory experiment and all authors contributed equally for the analyses of data and manuscript preparation.

Declaration of competing interests. The authors declare that they have no known competing financial interests or personal relationships that could have appeared to influence the work reported in this paper.

\section{REFERENCES}

[1] Alhamd, L., Hagihara, A. (2004): Litterfall of a subtropical evergreen broad-leaved forest in Okinawa Island, Japan. - Tropics 13(4): 255-268.

[2] Allen, S. E., Grimshaw, H. M., Parkinson, J. A., Quarmby, C. (1974): Chemical Analysis of Ecological Materials. - London: Blackwell Scientific Publication.

[3] Andrade, E. R., Jardim, J. G., Santos, B. A., Melo, F. P. L., Talora, D. C., Faria, D., Cazetta, E. (2015): Effects of habitat loss on taxonomic and phylogenetic diversity of understory Rubiaceae in Atlantic forest landscapes. - Forest Ecology and Management 349: 73-84.

[4] Aye, Y. Y., Pampasit, S., Umponstira, C., Thanacharoenchanaphas, K., Sasaki, N. (2014): Floristic composition, diversity and stand structure of tropical forests in Popa Mountain Park. - Journal of Environmental Protection 5: 1588-1602. http://dx.doi.org/10.4236/jep.2014.517150.

[5] Bhat, D. M., Naik, M. B., Patagar, S. G., Hedge, G. T., Kanade, Y. G., Hedge, G. N., Shastri, C. M., Shetti, D. M., Furtado, R. M. (2000): Forest dynamics in tropical rainforests of Uttara Kannada district in Western Ghats, India. - Current Science 79: 975-985.

[6] Binkley, D., Dunkin, K. A., DeBell, D., Ryan, M. G. (1992): Production and nutrient cycling in mixed plantations of Eucalyptus and Albizia in Hawaii. - Forest Science 38(2): 393-408.

[7] Bray, J. R., Gorham, E. (1964): Litter production in forests of the world. - Advances in Ecological Research 2: 101-157.

[8] Brockerhoff, E. G., Barbaro, L., Castagneyrol, B., Forrester, D. I., Gardiner, B., GonzálezOlabarria, J. R., Lyver, P. O’B., Meurisse, N., Oxbrough, A., Taki, H., Thompson, I. D., van der Plas, F., Jactel, H. (2017): Forest biodiversity, ecosystem functioning and the provision of ecosystem services. - Biodiversity and Conservation 26: 3005-3035. DOI: 10.1007/s10531-017-1453-2. 
[9] Carmo, A. B., Vasconcelos, H. L., Araújo, G. M. (2011): Estrutura da comunidade de plantas lenhosas em fragmentos de cerrado: relação com o tamanho do fragmento e seu nível de perturbação. - Revista Brasileira de Botanica 34(1): 31-38.

[10] Cayuela, L., Rey Benayas, J. M., Echeverría, C. (2006): Clearance and fragmentation of tropical montane forests in the Highlands of Chiapas, Mexico (1975-2000). - Forest Ecology and Management 226: 208-218.

[11] Chabrerie, O., Jamoneau, A., Gallet-Moron, E., Decocq, G. (2013): Maturation of forest edges is constrained by neighbouring agricultural land management. - Journal of Vegetation Science 24(1): 58-69.

[12] Champion, H. G., Seth, S. K. (1968): A revised survey of the forest types of India. - New Delhi (India): Government of India Press.

[13] Cochrane, M. A., Laurance, W. F. (2002): Fire as a large-scale edge effect in Amazonian forests. - Journal of Tropical Ecology 18(3): 311-325.

[14] Cuevas, E., Medina, E. (1988): Nutrient dynamics within Amazonian forests. II. Fine root growth, nutrient availability and leaf litter decomposition. - Oecologia 76: 222-235.

[15] Cuevas, E., Lugo, A. E. (1998): Dynamics of organic matter and nutrient return from litterfall in stands of ten tropical tree plantation species. - Forest Ecology and Management 112(3): 263-279.

[16] Debinski, D. M., Holt, R. D. (2000): Review: a survey and overview of habitat fragmentation experiments. - Conservation Biology 14(2): 342-355.

[17] Deng, C. Z., Hou, J. P., Li, S. C., Zhao, H. K., Fu, J. (1993): Study on forest litter in Ailao Mountain, North China. - Acta phytoecologia et geobotanica Sinica 17: 364-370. (in Chinese with English abstract).

[18] Didham, R. K. (1998): Altered leaf-litter decomposition rates in tropical forest fragments. - Oecologia 116: 397-406.

[19] Didham, R. K., Kapos, V., Ewers, R. M. (2012): Rethinking the conceptual foundations of habitat fragmentation research. - Oikos 121: 161-170.

[20] Echeverría, C., Newton, A. C., Lara, A., Benayas, J. M. R., Coomes, D. A. (2007): Impacts of forest fragmentation on species composition and forest structure in the temperate landscape of southern Chile. - Global Ecology and Biogeography 16: 426-439.

[21] Edwards, P. J. (1982): Studies of mineral cycling in a montane rain forest in New Guinea: V. Rates of cycling in throughfall and litter fall. - Journal of Ecology 70(3): 807-827.

[22] Epstein, E., Bloom, A. J. (2006): Mineral nutrition of plants: principles and perspectives. $2^{\text {nd }}$ edition. Sunderland, Sinauer Associates.

[23] Facelli, J. M., Pickett, S. T. A. (1991): Plant litter: its dynamics and effects on plant community structure. - The Botanical Review 57(1): 1-32.

[24] Fahrig, L. (2003): Effects of habitat fragmentation on biodiversity. - Annual Review of Ecology, Evolution, and Systematics 34: 487-515.

[25] Fahrig, L. (2013): Rethinking patch size and isolation effects: The habitat amount hypothesis. - Journal of Biogeography 40: 1649-1663.

[26] Feeley, K. J. (2004): The effects of forest fragmentation and increased edge exposure on leaf litter accumulation. - Journal of Tropical Ecology 20: 709-712.

[27] Feroz, S. M., Kabir, M. E., Hagihara, A. (2015): Species composition, diversity and stratification in subtropical evergreen broadleaf forests along a latitudinal thermal gradient in the Ryukyu Archipelago, Japan. - Global Ecology and Conservation 4: 63-72.

[28] Figueiredo-Filho, A., Moraes, G. F., Schaaf, L. B., Figueiredo, D. J. (2003): Seasonal assessment of litter deposition in a mixed rain forest located in the south of the state of Paraná. - Ciência Florestal 13: 11-18.

[29] Haase, R. (1999): Litterfall and nutrient return in seasonally flooded and non-flooded forest of the Pantanal, Mato Grosso, Brazil. - Forest Ecology and Management 117(1): 129-147.

[30] Haddad, N. M., Brudvig, L. A., Clobert, J., Davies, K. F., Gonzalez, A., Holt, R. D., Lovejoy, T. E., Sexton, J. O., Austin, M. P., Collins, C. D., Cook, W. M., Damschen, E. I., Ewers, R. M., Foster, B. L., Jenkins, C. N., King, A. J., Laurance, W. F., Levey, D. J., 
Margules, C. R., Melbourne, B. A., Nicholls, A. O., Orrock, J. L., Song, D., Townshend, J. R. (2015): Habitat fragmentation and its lasting impact on earth's ecosystem. - Applied Ecology 1(2): e1500052.

[31] Haridasan, K., Rao, R. R. (1987): Forest Flora of Meghalaya. - 2 vols. set, Bishen Singh Mahendrapal Singh, Dehra Dun.

[32] Harper, K., Macdonald, E. S., Burton, P. J., Chen, J. (2005): Edge influence on forest structure and composition in fragmented landscapes. - Conservation Biology 19(3): 768782.

[33] Hastwell, G. T., Facelli, J. M. (2000): Effects of leaf litter on woody seedlings in xeric successional communities. - Plant Ecology 148(2): 225-231. DOI:10.1023/A:1009834425538.

[34] Hobbs, R. J., Yates, C. J. (2003): Impacts of ecosystem fragmentation on plant populations: generalizing the idiosyncratic. - Australian Journal of Botany 51(5): 471-488.

[35] Hofmeister, J. K., Hosek, J., Brabec, M., Hedl, R., Modry, M. (2013): Strong influence of long-distance edge effect on herb-layer vegetation in forest fragments in an agricultural landscape. - Perspectives in Plant Ecology, Evolution and Systematics 15: 293-303.

[36] Ifo, S. A., Moutsambote, J., Koubouana, F., Yoka, J., Ndzai, S. F., Nucia, L., BouetouKadilamio, O., Mampouya, H., Jourdain, C., Bocko, Y., Mantota, A. B., Mbemba, M., Mouanga-Sokath, D., Odende, R., Mondzali, L. R., Wenina, Y. E. M., Ouissika, B. C., Joel, L. J. (2016): Tree species diversity, richness, and similarity in intact and degraded forest in the tropical rainforest of the Congo basin: case of the forest of Likouala in the Republic of Congo. - International Journal of Forestry Research 12: 7593681. https://doi.org/10.1155/2016/7593681.

[37] ISFR. (2019): India State of Forest Report 2019. - Ministry of Environment Forest and Climate Change. Dehradun.

[38] Kanjilal, V. N., Kanjilal, P. C., Das, A., De, R. N., Bor, N. L. (1940): Flora of Assam. - 5 vols. Government Press, Shillong.

[39] Kelty, M. J. (2006): The role of species mixtures in plantation forestry. - Forest Ecology and Management 233: 195-204.

[40] Kumar, Y., Syiemlieh, H. J., Singh, S. (2008): Vegetation cover and plant species of degraded landscape in the extremely wet Cherrapunji area, North-Eastern India. Transactions 30(2): 111-124.

[41] Lambert, A. M., Dudley, T. L., Robbins, J. (2014): Nutrient enrichment and soil conditions drive productivity in the large-statured invasive grass Arundo donax. - Aquatic Botany 112: $16-22$.

[42] Lamotte, S., Gajaseni, J., Malaisse, F. (1998): Structure diversity in three forest types of north-eastern Thailand (Sakaerat Reserve, Pak Tong Chai). - Biotechnology, Agronomy, Society and Environment 2(3): 192-202.

[43] Laurance, W. F. (1990): Comparative responses of five arboreal marsupials to tropical forest fragmentation. - Journal of Mammalogy 71(4): 641-653.

[44] Laurance, W. F., Bierregaard, R. O. (1997): Tropical forest remnants: ecology, management, and conservation of fragmented communities. - Chicago: University of Chicago Press.

[45] Laurance, W. F., Ferreira, L. V., Rankin-de Merona, J. M., Laurance, S. G., Hutchings, R., Lovejoy, T. E. (1998): Effects of forest fragmentation on recruitment patterns in Amazonian tree communities. - Conservation Biology 12: 460-464.

[46] Laurance, W. F., Cochrane, M. A. (2001): Synergistic effects in fragmented landscapes. Conservation Biology 15: 1488-1489.

[47] Laurance, W. F., Lovejoy, T. E., Vasconcelos, H. L., Bruna, E. M., Didham, R. K., Stouffer, P. C., Gascon, C., Bierregaard, R. O., Laurance, S. G., Sampiao, E. (2002): Ecosystem decay of Amazonian forest fragments: a 22-year investigation. - Conservation Biology 16: 605-618. 
[48] Laurance, W. F. (2004): Forest-climate interactions in fragmented tropical landscapes. Philosophical Transactions of the Royal Society of London B: Biological Sciences 359(1443): 345-352.

[49] Laurance, W. F., Peres, C. A. (2006): Emerging threats to tropical forests. - University of Chicago Press, Chicago.

[50] Laurance, W. F., Vasconcelos, H. L. (2009): Consequências ecológicas da fragmentação florestal na amazônia. - Oecologia Australis 13(3).

[51] Laurance, W. F., Camargo, J. L., Luizão, R. C., Laurance, S. G., Pimm, S. L., Bruna, E. M., Stouffer, P. C., Williamson, G. B., Benítez-Malvido, J., Vasconcelos, H. L., Van Houtan, K. S. (2011): The fate of Amazonian forest fragments: a 32-year investigation. Conservation Biology 144(1): 56-67.

[52] Lian, Y., Zhang, Q. (1998): Conversion of a natural broad-leafed evergreen forest into pure and mixed plantation forests in a subtropical area: effects on nutrient cycling. - Canadian Journal of Forest Research 28(10): 1518-1529.

[53] Liang, H. W. (1994): Studies on the litterfall of two forest types in mid-altitude of Laoshan mountain in Tianlin Country. - Chinese Journal of Ecology 13: 21-26.

[54] Lin, Y. M., He, J. Y., Yang, Z. W., Liu, C. D., Lin, P., Li, Z. J. (1999): The dynamics and production of litter falls of Castanopsis eyrei community in Wuyi Mountains. - Journal of Xiamen University Natural Sciences 38: 280-286.

[55] Lin, I., Cao, M. (2009): Edge effects on soil seed banks and understory vegetation in subtropical and tropical forests in Yunnan, SW China. - Forest Ecology and Management 257: 1344-1352.

[56] Lindsay, E. A., Cunningham, S. A. (2009): Livestock grazing exclusion and microhabitat variation affect invertebrates and litter decomposition rates in woodland remnants. - Forest Ecology and Management 258: 178-187.

[57] Lovejoy, T. E., Bierregaard, R. O., Rylands, A. B., Malcolm, J. R., Quintela, C. E., Harper, L. H., Brown, K. S., Powel, A. H. Jr., Powel, G. V. N., Schubart, H. O. R., Hays, M. B. (1986): Edge and other effects of isolation on Amazon forest fragments. - In: Soulé, M. E. (ed.) Conservation biology: the science of scarcity and diversity. Sunderland, Massachusetts: Sinauer.

[58] Magurran, A. E. (1988): Ecological diversity and its measurement. - Springer Netherlands.

[59] Majumdar, K., Shankar, U., Datta, B. K. (2014): Trends in tree diversity and stand structure during restoration: a case study in fragmented moist deciduous forest ecosystems of Northeast India. - Journal of Ecosystems, Article ID: 845142. http://dx.doi.org/10.1155/2014/845142.

[60] Melo, F. P. L., Lemire, D., Tabarelli, M. (2007): Extirpation of large-seeded seedlings from the edge of a large Brazilian Atlantic forest fragment. - Ecoscience 14(1): 124-129.

[61] Mir, A. H., Upadhaya, K. (2017): Effect of traditional management practices on woody species composition and structure in montane subtropical forests of Meghalaya, Northeast India. - Journal of Mountain Science 14(8): 1500-1512. http://dx.doi.org/10.1007/s11629016-4145-6.

[62] Misra, R. (1968): Ecology work book. - New Delhi (India): Oxford-IBH Publishing Company.

[63] Mittermeier, R. A., Robles-Gil, P., Hoffmann, M., Pilgrim, J. D., Brooks, T. B., Mittermeier, C. G., Lamoreux, J. L., Fonseca, G. A. B. (2004): Hotspots revisited: Earths biologically richest and most endangered ecoregions. - CEMEX, Mexico City, Mexico.

[64] Morellato, L. P. (1992): Nutrient cycling in two south-east Brazilian forests. I Litterfall and litter standing crop. - Journal of Tropical Ecology 1: 205-215.

[65] Mueller-Dombois, D., Ellenberg, H. (1974): Aims and Methods of Vegetation Analysis. New York (USA): John Wiley and Sons.

[66] Murcia, C. (1995): Edge effects in fragmented forests: implications for conservation. Trends in Ecology and Evolution 10: 58-62.

https://doi.org/10.1016/S0169-5347(00)88977-6PMID:21236953. 
[67] Murphy, P. G., Lugo, A. E. (1986): Ecology of tropical dry forest. - Annual Review of Ecology and Systematics 17(1): 67-88.

[68] Naidu, M. T., Kumar, O. A. (2016): Tree diversity, stand structure, and community composition of tropical forests in Eastern Ghats of Andhra Pradesh, India. - Journal of Asia Pacific Biodiversity 9: 328-334.

[69] Norgrove, L., Hauser, S. (2000): Leaf properties, litter fall, and nutrient inputs of Terminalia ivorensis at different tree stand densities in a tropical timber-food crop multistrata system. - Canadian Journal of Forest Research 30(9): 1400-1409.

[70] Ochoa-Gaono, S., Gonzalez-Espinosa, M., Meave, J. A., Sorani-Dal Bon, V. (2004): Effect of fragmentation on the woody flora of the highlands of Chiapas, Mexico. - Biodiversity and Conservation 13: 867-884.

[71] Odiwe, A. I., Muoghalu, J. I. (2003): Litterfall dynamics and forest floor litter as influenced by fire in a secondary lowland rain forest in Nigeria. - Tropical Ecology 44(2): 243-251.

[72] Oliveira, M. A., Santos, A. M. M., Tabarelli, M. (2008): Profound impoverishment of the large-tree stand in a hyper fragmented landscape of the Atlantic forest. - Forest Ecology and Management 256(11): 1910-1917.

[73] Pao, N. T., Upadhaya, K. (2017): Effect of fragmentation and anthropogenic disturbances on floristic composition and structure of subtropical broad leaved humid forest in Meghalaya, northeast India. - Applied Ecology and Environmental Research 15(4): 385407. https://doi.org/10.15666/ aeer/1504_385407.

[74] Pao, N. T., Upadhaya, K. (2019): Seasonal dynamics of soil microbial biomass in fragmented patches of subtropical humid forest of Jaintia hills in Meghalaya, Northeast India. - Forest Systems 28(1): e002. https://doi.org/10.5424/fs/2019281-13972.

[75] Parthasarathy, N., Kinhal, V., Praveenkumar, L. (1992): Plant species diversity and human impacts in the tropical wet evergreen forests of southern Western Ghats. - In: Proceedings of the Indo-French Workshop on Tropical Forest Ecosystems: Natural Functioning and Anthropogenic Impact, French Institute, Pondicherry, India.

[76] Pinto, S. I. C., Martins, S. V., Barros, N. F., Dias, H. C. T. (2008): Litter production in two successional stages of semideciduous seasonal forest in the Mata do Paraíso Reserve, in Viçosa, MG. - Revista Árvore 32: 545-556.

[77] Prescott, C. E., Vesterdal, L., Simard, S. W., Preston, C. M. (2004): Influence of initial chemistry on decomposition of foliar litter in contrasting forest types in British Columbia. - Canadian Journal of Forest Research 34: 1714-1729.

[78] Prescott, C. E. (2010): Litter decomposition: what controls it and how can we alter it to sequester more carbon in forest soils? - Biogeochemistry 101: 133-149. DOI 10.1007/s10533-010-9439-0.

[79] Proctor, J. (1987): Nutrient cycling in primary and old secondary rain forests. - Applied Geography 7: 135-152.

[80] Rosati, L., Fipaldini, M., Marignani, M., Blasi, C. (2010): Effects of fragmentation on vascular plant diversity in a Mediterranean forest archipelago. - Plant Biosystems 144(1): 38-46.

[81] Rubinstein, A., Vasconcelos, H. L. (2005): Leaf-litter decomposition in Amazonian forest fragments. - Journal of Tropical Ecology 21: 699-702.

[82] Sala, O. E., Chapin III, F. S., Armesto, J. J., Berlow, E., Bloomfield, J., Dirzo, R., HuberSanwald, E., Huenneke, L. F., Jackson, R. B., Kinzig, A., Leemans, R., Lodge, D. M., Mooney, H. A., Oesterheld, M., Poff, N. L., Sykes, M. T., Walker, B. H., Walker, M., Wall, D. H. (2000): Global biodiversity scenarios for the year 2100. - Science 287: 1770-1774.

[83] Santos, K. D., Kinoshita, L. S., Flavio, A. M., Santos, D. (2007): Tree species composition and similarity in semideciduous forest fragments of southeastern Brazil. - Biological Conservation 135: 268-277.

[84] Santos, B. A., Peres, C. A., Oliveira, M. A., Grillo, A., Alves-Costa, C. P., Tabarelli, M. (2008): Drastic erosion in functional attributes of tree assemblages in Atlantic forest fragments of northeastern Brazil. - Biological Conservation 141(1): 249-260. 
[85] Saunders, D. A., Hobbs, R. J., Margules, C. R. (1991): Biological consequences of ecosystem fragmentation: a review. - Conservation Biology 5: 18-32.

[86] Schmidt, M. (2019): Fragmentation of landscapes: modelling ecosystem services of transition zones. - Doctoral dissertation, University of Potsdam.

[87] Singh, J. S., Raghubansh, A. S., Singh, R. S., Srivastava, S. C. (1989): Microbial biomass acts as a source of plant nutrients in dry tropical forest and savanna. - Nature 338(6215): 499-500. http://www.researchgate.net/publication/236941524.

[88] Sonnier, G., Jamonaeu, A., Decocq, G. (2014): Evidence for a direct negative effect of habitat fragmentation on forest herb functional diversity. - Landscape Ecology 29: 857866.

[89] Spain, A. V. (1984): Litter fall and the standing crop of litter in three tropical Australian rain forests. - Journal of Ecology 72: 947-961.

[90] Sundarapandian, S. M., Swamy, P. S. (1999): Litter production and leaf-litter decomposition of selected tree species in tropical forests at Kodayar in the Western Ghats, India. - Forest Ecology and Management 123(2): 231-244.

[91] Swift, M. J., Russell-Smith, A., Perfect, T. J. (1981): Decomposition and mineral-nutrient dynamics of plant litter in a regenerating bush-fallow in sub-humid tropical Nigeria. Journal of Ecology 69(2): 981-995.

[92] Tabarelli, M., Mantovani, W., Peres, C. A. (1999): Effects of habitat fragmentation on plant guild structure in the montane Atlantic forest of southeastern Brazil. - Biological Conservation 91: 119-128.

[93] Tang, J. W., Cao, M., Zhang, J. H., Li, M. H. (2010): Litterfall production, decomposition and nutrient use efficiency varies with tropical forest types in Xishuangbanna, SW China: a 10-year study. - Plant Soil 335(1-2): 271-288.

[94] Tian, D. L., Zhao, K. (1989): Studies on the litter in a Chinese fir plantation ecosystem II. Nutrient contents and decomposition rate of litter. - Journal of Central-South Forestry College 9: 45-55. (in Chinese with English abstract).

[95] Tripathi, O. P., Upadhaya, K., Tripathi, R. S., Pandey, H. N. (2010): Diversity, dominance and population structure of tree species along fragment-size gradient of a subtropical humid forest of northeast India. - Research Journal of Environmental and Earth Sciences 2(2): 97 105.

[96] Tu, M. Z., Yao, W. H., Weng, H., Li, Z. A. (1993): Characteristics of litter in evergreen broadleaved forest of the Dinghu mountain. - Acta Pedologica Sinica 30: 34-42. (Chinese with English abstract).

[97] Tuomisto, H., Ruokolainen, K., Yli-Halla, M. (2003): Dispersal, environment, and floristic variation of Western Amazonian forests. - Science 299: 241-244.

[98] Turner, I. M., Corlett, R. T. (1996): The conservation value of small, isolated fragments of lowland tropical rain forest. - Tree 11(8): 330-333.

[99] Upadhaya, K., Pandey, H. N., Law, P. S., Tripathi, R. S. (2003): Tree diversity in sacred groves of the Jaintia hills in Meghalaya, northeast India. - Biodiversity and Conservation 12: 583-597.

[100] Upadhaya, K., Chaudhury, G., Sarmah, K. (2014): Anthropogenic threats and plant diversity conservation in Cherrapunji- one of the wettest places on Earth. - Keanean Journal of Science 3: 1-18.

[101] Upadhaya, K. (2015): Structure and floristic composition of subtropical broad-leaved humid forest of Cherapunjee in Meghalaya, Northeast India. - Journal of Biodiversity Management and Forestry 4: 4. http://dx.doi.org/2327-4417.1000149.

[102] Valappil, N. I., Swarupanandan, K. (1996): Regeneration dynamics and sylvigenesis in the moist deciduous forests of southwest India. - New Forest 11(3): 185-205.

[103] Vasconcelos, H. L., Luizão, F. (2004): Litter production and litter nutrient concentrations in a fragmented Amazonian landscape. - Ecological Application 14: 884-892. 
[104] Vasconcelos, H. L., Laurance, W. F. (2005): Influence of habitat, litter type, and soil invertebrates on leaf-litter decomposition in a fragmented Amazonian landscape. Oecologia 144: 456-462.

[105] Vitousek, P. M. (1984): Litterfall, nutrient cycling, and nutrient limitation in tropical forests. - Ecology 65: 285-298.

[106] Vitousek, P. M., Sanford Jr, R. L. (1986): Nutrient cycling in moist tropical forest. - Annual Review of Ecology and Systematics 17(1): 137-167.

[107] Vitousek, P. M., Turner, D. R., Kitayama, K. (1995): Foliar nutrients during long-term soil development in Hawaiian montane rain forest. - Ecology 76(3): 712-720.

[108] Vitousek, P. M. (2004): Nutrient cycling and limitation: Hawaii as a model system. Princeton University Press.

[109] Vogt, K. A., Grier, C. C., Vogt, D. J. (1986): Production, turnover and nutrient dynamics of above- and belowground detritus of world forests. - Advances in Ecological Research 15: 303-377.

[110] Weng, H., Li, Z. A., Tu, M. Z., Yao, W. H. (1993): The production and nutrient contents of litter in forest of Dinghushan. - Acta Phytoecologica et Geobotanica Sinica 17: 299-304. (in Chinese with English abstract).

[111] White, D. L. (1988): Litter decomposition in southern Appalachian black locust and pinehardwood stands: litter quality and nitrogen dynamics. - Canadian Journal of Forest Research 18: 54-63.

[112] Wilcove, D. S., McLellan, C. H., Dobson, A. P. (1986): Habitat fragmentation in the temperate zone. - In: Soule, M. E. (ed.) Conservation Biology. Sunderland, M.A. Sinauer.

[113] Wilson, M. C., Chen, X-Y., Corlett, R. T., Didham, R. K., Ding, P., Holt, R. D., Holyoak, M., Hu, G., Hughes, A. C., Jiang, L., Laurance, W. F., Liu, J., Pimm, S. L., Robinson, S. K., Russo, S. E., Si, X., Wilcove, D. S., Wu, J., Yu, M. (2016): Habitat fragmentation and biodiversity conservation: key findings and future challenges. - Landscape Ecology 31: 219-227.

[114] Wu, J. G. (2013): Key concepts and research topics in landscape ecology revisited: 30 years after the Allerton Park workshop. - Landscape Ecology 28: 1-11.

[115] Yang, Y. S., Li, Z. W., Liu, A. Q. (1993): Studies on soil fertility for natural forest of Castanopsis kawakamii replaced by broadleaf plantation. - Journal of Northeast Forestry University 21: 14-21. (in Chinese with English abstract).

[116] Yang, Y. S., Guo, J. F., Chen, G. S., Xie, J. S., Cai, L. P., Lin, P. (2004): Litterfall, nutrient return, and leaf-litter decomposition in four plantations compared with a natural forest in subtropical China. - Annals of Forest Science 61(5): 465-476.

[117] Zhang, H., Yuan, W., Dong, W., Liu, S. (2014): Seasonal patterns of litterfall in forest ecosystem worldwide. - Ecological Complexity 20: 240-247.

[118] Zheng, W. J., Shao, C., Wang, L. M., Lin, P. (1995): Dynamics of nutrient elements in litterfall of subtropical rain forest of Hexi in Fujian. - Journal of Tropical and Subtropical Botany 3: 38-43. (in Chinese with English abstract).

[119] Zhou, J., Lang, X., Du, B., Zhang, H., Liu, H., Zhang, Y., Shang, L. (2016): Litterfall and nutrient return in moist evergreen broad-leaved primary forest and mixed subtropical secondary deciduous broad-leaved forest in China. - European Journal of Forest Research 135(1): 77-86. 